\title{
6 Automatisierung, öffentlicher Verkehr und Mobility as a Service: Erfahrungen aus Tests mit automatisierten Shuttlebussen
}

Aggelos Soteropoulos, Emilia M. Bruck, Martin Berger, Alexander Egoldt, Arne Holst, Thomas Richter, Zoltán László

1. Einleitung

2. Öffentlicher Verkehr: Derzeitige Angebotsformen und zukünftige Use Cases automatisierter Fahrzeuge

3. Automatisierte Shuttlebusse als vorrangiger Use Case von Testprojekten im öffentlichen Verkehr

4. Technische und rechtliche Aspekte beim Testen automatisierter Shuttlebusse im öffentlichen Verkehr

4.1 Der verwendete Shuttlebus

4.2 Streckenfindung, Infrastrukturanpassung und Orientierung des Shuttlebusses

4.3 Zulassung und Inbetriebnahme

4.4 Bisherige Erkenntnisse

5. Betrieb und Integration automatisierter Shuttlebusse in den öffentlichen Verkehr

5.1 Verwendeter Shuttlebus und Betrieb

5.2 Integration in Kundeninformationssysteme des öffentlichen Verkehrs 96

5.3 Bisherige Erkenntnisse

$\begin{array}{llr}\text { 6. Fazit } & 100\end{array}$

Literatur

Aggelos Soteropoulos

TU Wien, future.lab Research Center und Forschungsbereich Verkehrssystemplanung (IVS) aggelos.soteropoulos@tuwien.ac.at

Emilia M. Bruck

TU Wien, future.lab Research Center und Forschungsbereich Örtliche Raumplanung (IFOER) emilia.bruck@tuwien.ac.at

Martin Berger

TU Wien, Forschungsbereich Verkehrssystemplanung (IVS)

martin.kp.berger@tuwien.ac.at 


\author{
Alexander Egoldt \\ TU Berlin, Fachgebiet Straßenplanung und Straßenbetrieb \\ a.egoldt@spb.tu-berlin.de \\ Arne Holst \\ TU Berlin, Fachgebiet Straßenplanung und Straßenbetrieb \\ a.holst@spb.tu-berlin.de \\ Thomas Richter \\ TU Berlin, Fachgebiet Straßenplanung und Straßenbetrieb \\ t.richter@spb.tu-berlin.de \\ Zoltán László \\ Schweizerische Bundesbahnen (SBB) , Lead On-Demand Mobilität , Neue Mobilitätsdienstleistungen \\ zoltan.laszlo@sbb.ch
}

\title{
1. EINLEITUNG
}

Automatisiertes Fahren verändert die zukünftige Mobilität grundlegend und wirkt sich auch auf den öffentlichen Verkehr (ÖV) aus. Häufig wird hierbei von einer weiteren Verschiebung der Grenzen zwischen dem klassischen ÖV und dem motorisierten Individualverkehr (MIV) und von einem Übergangsbereich des öffentlichen Individualverkehrs (ÖIV) mit automatisierten Fahrzeugen bzw. von einer Individualisierung des öffentlichen Verkehrs gesprochen (vgl. Lenz/ Fraedrich 2015: 189; Röhrleef 2017: 15; Bruns et al. 2018: 12; Barillère-Scholz et al. 2020: 16): Bereits heute differenziert sich das Mobilitätsangebot durch neue Angebotsformen wie beispielsweise Car-Sharing oder Ride-Hailing aus.

Neue Mobilitätsdienstleister bieten insbesondere in Städten bedarfsgerechte, individualisierte Verkehrsmöglichkeiten, sogenannte On-Demand-Mobilität, an und erweitern damit das Mobilitätsangebot, was vor dem Hintergrund der zunehmenden Digitalisierung weiter zunehmen wird (vgl. Barillère-Scholz et al. 2020: 15; Buffat et al. 2018: 90; Lenz/Fraedrich 2015: 183). Mit der technologischen Entwicklung des automatisierten Fahrens bieten sich Möglichkeiten, noch nicht da gewesene Geschäftsmodelle zu entwickeln, die weiteren Anbietenden den Markteintritt eröffnen: Durch die Automatisierung sind disruptive Entwicklungen im Mobilitätsbereich und eine weitere Transformation der heute bekannten Angebotsformen vorstellbar (vgl. Gertz/ Dörnemann 2016: 5). Hierbei nimmt ebenso die Bedeutung von Mobility as a Service (MaaS) zu, also öffentliche und private Verkehrsangebote sowie unterschiedliche Verkehrsarten mittels einheitlichem, digitalem Zugangsportal (Plattform, App) zu kombinieren und so auf individuelle Bedürfnisse angepasste, maßgeschneiderte Mobilitätslösungen anzubieten (vgl. EPOMM 2017; Jittrapirom et al. 2017: 14).

Gleichfalls werden mit dem automatisierten Fahren im öffentlichen Verkehr auch Potentiale einer verbesserten Wirtschaftlichkeit des öffentlichen Verkehrs verbunden, falls Personalkosten verrin- 
gert werden können: Wenn kein/e Fahrerln mehr benötigt wird und neue ergänzende Angebotsformen in Form kleinerer, flexiblerer Einheiten eingesetzt sowie Fahrzeugkonzepte zunehmend bedarfsgerecht genutzt werden, bietet sich die Chance für einen wirtschaftlicheren, effizienteren und nachfragegerechteren Einsatz von Mobilitätsangeboten (vgl. Hörl 2020: 2; Hörl et al. 2019: 60; Bösch et al. 2018: 7; Gertz/Dörnemann 2016: 22) - auch wenn zum Teil neue bzw. zusätzliche Kosten beispielsweise für die Dispositionssysteme oder zusätzliches Personal für Reparatur und Reinigung der Fahrzeug entstehen (vgl. Bruns et al. 2018: 5). Darüber hinaus ermöglichen die neuen Angebotsformen im Gegensatz zu den derzeit häufig bestehenden, oftmals langfristigen Konzessionen, die meist keine maßgeblichen Veränderungen und Anpassungen während der Laufzeit erlauben, die Möglichkeit, sich angebotsseitig stärker an den individuellen Bedürfnissen der Menschen zu orientieren, spezifische Anpassungen vorzunehmen und so den öffentlichen Verkehr zu attraktivieren und zu stärken (Barillère-Scholz et al. 2020: 15).

Abbildung 1: Verschiebung der Grenzen zwischen öffentlichem Verkehr und motorisiertem Individualverkehr

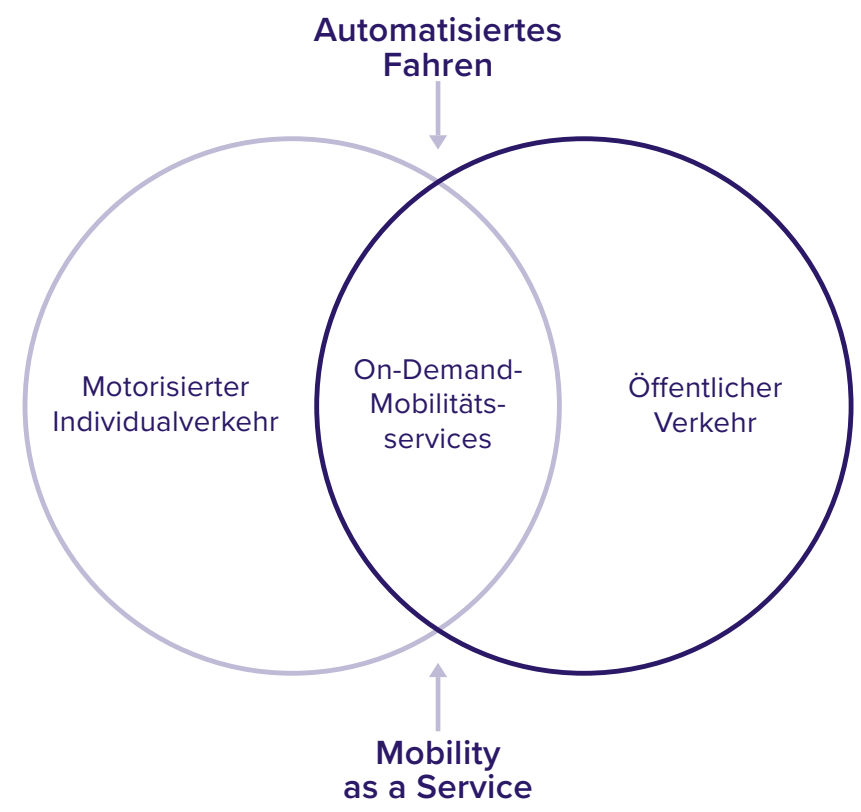

Quelle: eigene Darstellung

Aktuell finden sich zahlreiche Testprojekte zum automatisierten Fahren im öffentlichen Verkehr, in deren Rahmen automatisierte Technologien in ausgewählten Anwendungsfällen frühzeitig in der Praxis eingesetzt und neue Verkehrsangebote geschaffen sowie ganzheitliche Fahrzeugkonzepte entwickelt werden. Diese Testprojekte stellen sicher, dass der operative Betrieb von On-Demand-Mobilitätsangeboten, die datengestützte Verkehrsanalyse sowie die Plattformlösungen schon heute für die automatisierte Mobilität von morgen bereit sind. Hier geht es darum, perspektivisch Systembausteine bereitzustellen, um es Kundlnnen wie Verkehrsanbietern, Gemeinden und Städten zu ermöglichen, neue Mobilitätsformen im öffentlichen Verkehr zu betreiben (vgl. Barillère-Scholz et al. 2020: 18).

Die Testprojekte zum automatisierten Fahren im öffentlichen Verkehr konzentrieren sich derzeit insbesondere im deutschsprachigen Raum mehrheitlich auf die Erprobung von automatisierten Shuttlebussen. Diese kommen dabei auf einer eigens genehmigten, festen Route zum Einsatz und werden mehrheitlich noch durch Sicherheitsfahrerlnnen bzw. Operatorlnnen an Bord begleitet. Im Fokus der Erprobung der automatisierten Shuttlebusse stehen in der 
Mehrzahl der Projekte Aspekte der technologischen, organisatorischen und betrieblichen sowie der wirtschaftlichen Machbarkeit (vgl. Jürgens 2020).

Dieser Beitrag thematisiert anhand von Erfahrungen aus beispielhaften Testprojekten mit automatisierten Shuttlebussen speziell die Aspekte (1) der technologischen Machbarkeit bzw. der Befahrbarkeit von Straßenräumen sowie (2) der Integration der automatisierten Shuttlebusse in das bestehende ÖV-Angebot.

Hierzu werden zunächst die Relevanz der Automatisierung für den öffentlichen Verkehr dargelegt und verschiedene Use Cases des automatisierten Fahrens im öffentlichen Verkehr aufgezeigt. Danach beleuchtet der Beitrag speziell den Use Case des automatisierten Shuttlebusses und gibt einen Überblick der verschiedenen Tests mit automatisierten Shuttlebussen in Europa und insbesondere im deutschsprachigen Raum.

Anschließend werden am Beispiel des Projekts "autoNV OPR“ in Ostprignitz-Ruppin in Deutschland die technischen und rechtlichen Aspekte beim Test mit automatisierten Shuttlebussen aufgezeigt und anhand des Projekts "MyShuttle“ in Zug in der Schweiz die Erfahrungen hinsichtlich des Betriebs und der Integration des Shuttlebusses in das bestehende öffentliche Verkehrssystem näher beleuchtet. Abschließend erfolgt eine Zusammenfassung der wesentlichen Erkenntnisse aus beiden Beispielprojekten sowie eine Ableitung planerischer und politischer Implikationen.

\section{2. ÖFFENTLICHER VERKEHR: DERZEITIGE ANGEBOTSFORMEN UND ZUKÜNFTIGE USE CASES AUTOMATISIERTER FAHRZEUGE}

Grundsätzlich fallen unter den öffentlichen Verkehr alle Angebote zur regelmäßigen und gewerbsmäßigen Personenbeförderung. Diese zeichnen sich vor allem durch eine geteilte und für jedermann unter gleichen Bedingungen zugängliche Nutzung aus (vgl. Hörold 2016: 38; Bruns et al. 2018: 15). Da die Bereitstellung des ÖV-Angebotes zumeist öffentliche Aufgabe im Rahmen der Daseinsvorsorge für Mobilität ist (vgl. Rollinger/Amtmann 2009: 6), sind die ÖVAngebote im Betrieb meist nicht vollständig kostendeckend und erhalten demnach öffentliche Zuschüsse. Öffentliche Verkehrsangebote zeichnen sich zudem durch folgende Merkmale aus, die meist rechtlich verankert sind und dadurch die bekannten, heute weitestgehend fixen Elemente des ÖV wie Abfahrtszeiten, Haltestellen und Linienführung aufweisen (vgl. Bruns et al. 2018):

- Ausführung durch berechtigte Verkehrsunternehmen auf konzessionierten Linien beziehungsweise Routen

- Fahrplanpflicht: Aufstellung und Publikation eines Fahrplans

- Betriebspflicht: Durchführung des publizierten Angebots unabhängig von den äußeren Bedingungen und der momentanen Nachfrage

- Tarifpflicht: Fixierung und Veröffentlichung von Beförderungsbedingungen und Fahrpreisen 
Zusätzlich zu dem eher klassischen Linienverkehr finden sich jedoch auch Angebotsformen, die durch eine Flexibilisierung der Abfahrtszeit (On-Demand-Verkehr) und der Route/Linie, variable Halte (ohne ortsfeste Haltestellen) oder eine Kombination dieser Elemente stärker an den individuellen Bedürfnissen der Fahrgäste orientiert sind (vgl. Bruns et al. 2018: 15). Hierbei lassen sich flexible Angebotsformen, d. h. Mikro-ÖV-Systeme oder Bedarfsverkehre, und alternative Angebotsformen wie das Car-, oder Ride-Sharing unterscheiden. Sommer (2018: 3f.) spricht in diesem Zusammenhang von öffentlich zugänglichen Angeboten eines „erweiterten“ öffentlichen Verkehrs. Tabelle 1 gibt einen Überblick der verschiedenen derzeitigen Angebotsformen des öffentlichen Verkehrs. Es ist jedoch durchaus auch üblich, dass Elemente der flexiblen Angebotsformen und jene der alternativen Angebotsformen (z. B. Anmeldung der Nutzung etc.) kombiniert werden.

Tabelle 1: Überblick der Merkmale der unterschiedlichen Angebotsformen im öffentlichen Verkehr

\section{Klassischer \\ Linienverkehr}

\section{Flexible Angebotsformen}

\section{Alternative Angebotsformen}

\begin{tabular}{|c|c|c|c|}
\hline $\begin{array}{l}\text { Eigen- } \\
\text { schaften }\end{array}$ & $\begin{array}{l}\text { Festgelegte Bedienung, } \\
\text { Fahrplan und eindeutiger } \\
\text { Linienweg }\end{array}$ & $\begin{array}{l}\text { Für den Einsatz in Zeiten und } \\
\text { Räumen schwacher Nachfrage }\end{array}$ & $\begin{array}{l}\text { In der Regel genehmigungsfrei und } \\
\text { keine Beförderungsgarantie, einmalige } \\
\text { Registrierung bzw. Anmeldung erforderlich } \\
\text { (außer sozialer Fahrdienst) }\end{array}$ \\
\hline Beispiele & $\begin{array}{l}\text { - Übergeordnetes Bahn- } \\
\text { Bus-Grundnetz, } \\
\text { - lokaler Linienverkehr zur } \\
\text { Erschließung }\end{array}$ & $\begin{array}{l}\text { Mit Fahrplan: } \\
\text { - } \quad \text { linienförmiges Bedienungsge- } \\
\text { biet (Bedarfslinienverkehr) } \\
\text { - } \quad \text { korridorförmiges Bedienungsge- } \\
\text { biet (i. d. R. zwei feste Haltestel- } \\
\text { len): Richtungsbandbetrieb (z. B. } \\
\text { Rufbus) } \\
\text { sektorförmiges Bedienungs- } \\
\text { gebiet (ein Verknüpfungspunkt): } \\
\text { Sektorbetrieb (z. B. Anruf-Sam- } \\
\text { meltaxi, Zubringer) } \\
\text { Ohne Fahrplan: } \\
\text { flächenförmiges Bedienungsge- } \\
\text { biet und mit Fahrtenbündelung } \\
\text { (Flächenbetrieb bzw. Flächen- } \\
\text { bedienung) }\end{array}$ & 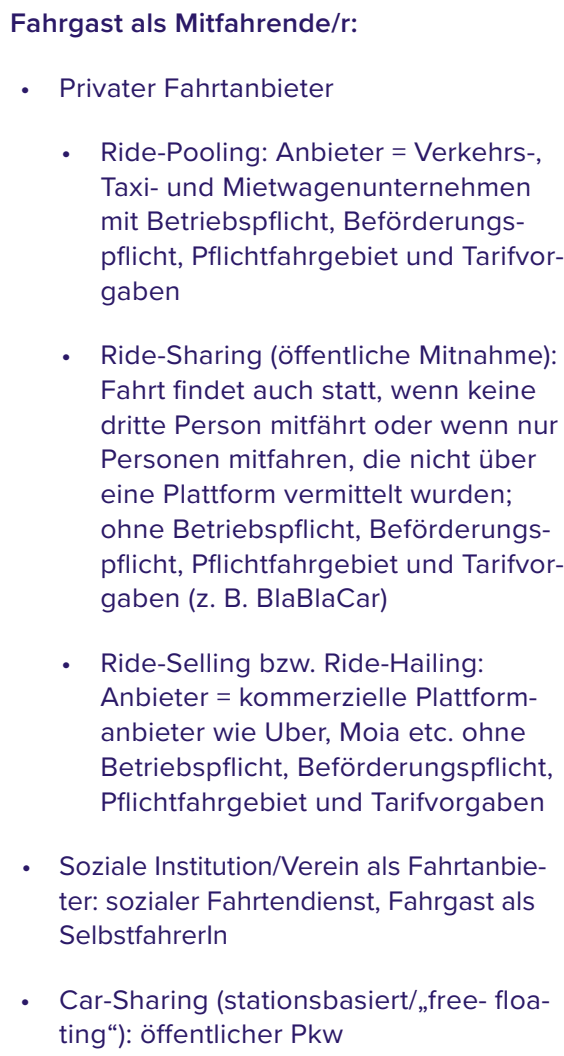 \\
\hline $\begin{array}{l}\text { Verkehrs- } \\
\text { mittel }\end{array}$ & $\begin{array}{l}\text { U-Bahn, S-Bahn/ } \\
\text { Regionalbahn, Straßenbahn, } \\
\text { Gelenkbus, Standardbus, } \\
\text { Minibus (Bürgerbus), } \\
\text { Shuttlebus }\end{array}$ & $\begin{array}{l}\text { Standardbus, Rufbus, Minibus, } \\
\text { Shuttlebus, Van, Anruf-Sammeltaxi }\end{array}$ & Sammeltaxi/Van/Pkw, Einzeltaxi/Pkw \\
\hline
\end{tabular}


Die Erweiterung und Kombination des klassischen Linienverkehrs durch flexible und alternative und hierbei auch private Angebotsformen wird derzeit vor allem im Kontext von Mobilitätsdienstleistungen (MaaS) diskutiert. Die Kombination findet dabei mittels einheitlichem, digitalem Zugangsportal (z. B. Plattform, App) statt. Das Mobilitätsangebot ermöglicht das Zusammenspiel unterschiedlicher Modi, die den jeweiligen persönlichen Bedürfnissen am besten gerecht werden - es werden also individuell maßgeschneiderte Mobilitätslösungen angeboten (vgl. EPOMM 2017; Jittrapirom et al., 2017: 14). Durch MaaS - so die Idee - sollen die Effizienz von bestehenden Mobilitätssystemen sowie die öffentlichen Ressourcen verbessert werden (vgl. Hoadley 2017: 5ff.).

Automatisiertes Fahren bietet in Anbetracht der bestehenden Angebotsformen im erweiterten öffentlichen Verkehr Optionen für eine weitere Ausdifferenzierung des Angebots bzw. Möglichkeiten für die Neugestaltung der Intermobilität, für eine weitere Flexibilisierung und Individualisierung sowie für die zeitliche und räumliche Verdichtung des Angebotes (vgl. Lenz/ Fraedrich 2015: 189). Ermöglicht wird dies durch den Wegfall von Personalkosten, die im öffentlichen Verkehr einen hohen Anteil der Gesamtkosten ausmachen (vgl. Hell 2006: 169). Durch den Wegfall bzw. die Reduktion der Personalkosten könnten die Angebote wirtschaftlicher betrieben werden (vgl. Hörl 2020: 2; Hörl et al. 2019: 60; Bösch et al. 2018: 7; Gertz/ Dörnemann 2016: 22). Ungeklärt ist jedoch, inwieweit dennoch Begleitpersonal benötigt wird, um die Sicherheit der Fahrgäste sicherzustellen (vgl. Salonen/Haavisto 2019: 13; Mitteregger et al. 2019: 610).

Die fortschreitende Automatisierung könnte zu neuen Angebotsformen bzw. Verkehrsmitteln führen (vgl. Soteropoulos et al. 2019: 104). Dies umfasst sowohl den klassischen Linienverkehr (z. B. AV-Standardbus, AV-Gelenkbus), die flexiblen Angebotsformen (z. B. AV-Minibus/ Shuttlebus, AV-Van/AV-Ride-Sharing) und die alternativen Angebotsformen (z. B. AV-Sammeltaxi/AV-Ride-Sharing, AV-Einzeltaxi/AV-Car-Sharing; vgl. Bruns et al. 2018: 21). Im klassischen Linienverkehr werden auch unter AV-Bedingungen große Fahrzeuge verkehren, mit denen das Mindestangebot der öffentlichen Hand abgedeckt werden wird, während die flexiblen und alternativen Angebotsformen eher mit kleinen Fahrzeugen bedient werden. Ihre Marktnischen ergeben sich durch zusätzliche Angebote bzw. Angebote zur Kombination mit dem Linienverkehr auf der ersten und letzten Meile, insbesondere in Zeiten und Räumen schwacher Nachfrage (vgl. Ohnemus/Perl 2016: 591).

Tabelle 2 (auf der nächsten Seite) gibt einen Überblick zu den Merkmalen verschiedener Angebotsformen bzw. Verkehrsmittel im erweiterten öffentlichen Verkehr unter Berücksichtigung der Automatisierung im Straßenverkehr. Der automatisierte Shuttlebus nimmt hierbei eine Sonderstellung ein, da er hinsichtlich seiner Fahrzeuggröße sowohl im Linienbetrieb auf einer festen Route mit fixem Zeitplan und fixen Haltestellen eingesetzt werden könnte als auch in flexibler Form je nach Bedarf und mit Halten on demand.

Speziell bei den möglichen flexiblen und alternativen Angebotsformen mit Automatisierung zeigt sich, dass diese für unterschiedliche Bedienungsformen infrage kommen könnten (siehe Tab. 3). So eignet sich der (AV-)Minibus bzw. -Shuttlebus am ehesten für den Linienbetrieb oder als Rufbus mit Richtungsbandbetrieb. Der (AV-)Van (Ride-Sharing) und das (AV-)Sammeltaxi (Ride-Sharing) eignen sich hingegen am ehesten als Anruf-Sammeltaxi, als Zubringer, d.h. für den Sektorbetrieb, oder für den Flächenbetrieb bzw. für die Flächenbedienung. Auch das (AV-)Einzeltaxi (Car-Sharing) eignet sich am ehesten für den Sektor- sowie den Flächenbetrieb.

Insgesamt zeigen sich somit prinzipiell vielfältige Einsatzmöglichkeiten für automatisierte Fahrzeuge im öffentlichen Verkehr. In Zukunft müssen diese unterschiedlichen Fahrzeugkonzepte ideal eingesetzt werden. Um deren bestmöglichen Einsatz bereits heute auszuloten, 


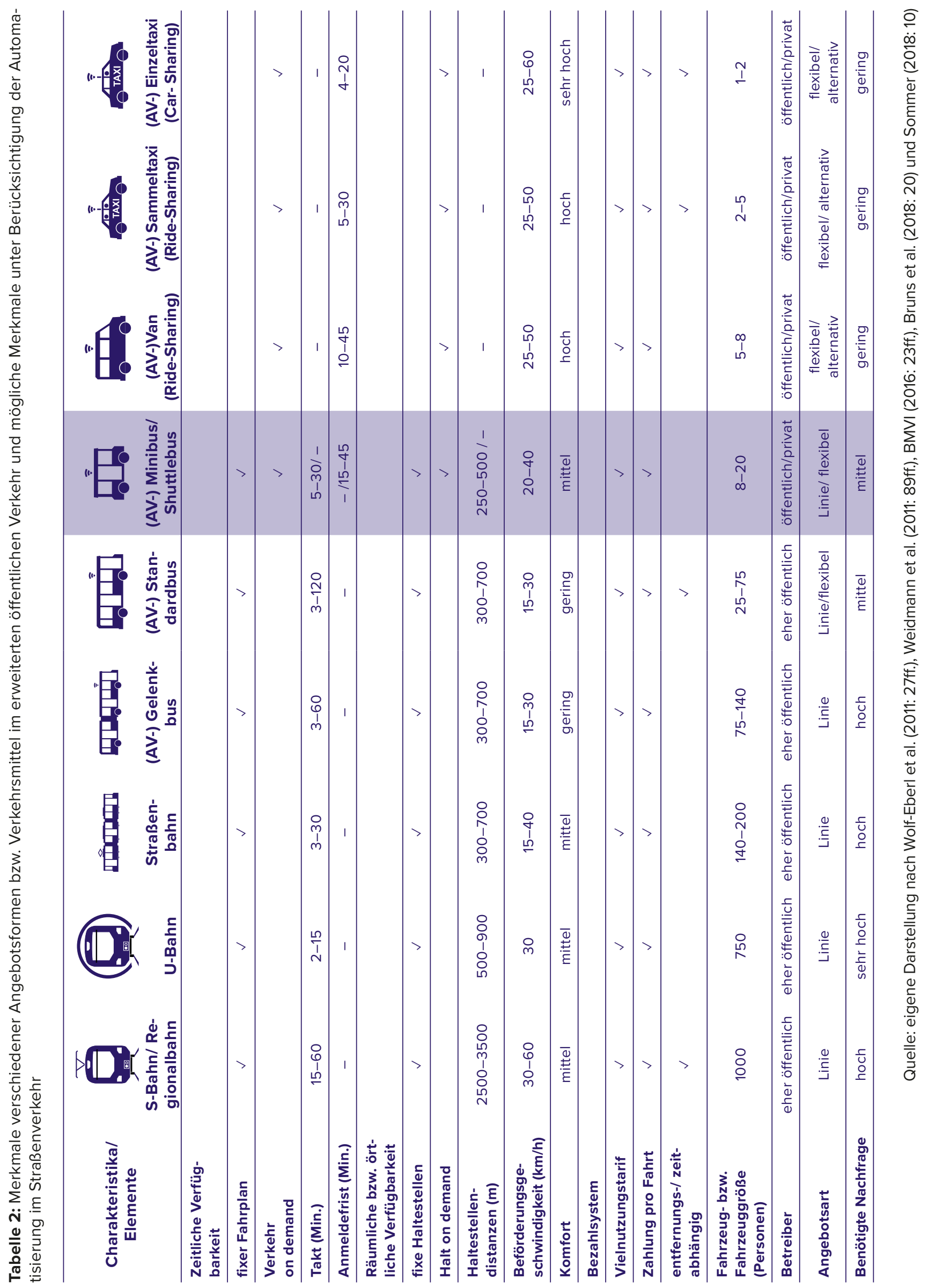


finden sich derzeit bereits zahlreiche Testprojekte zum automatisierten Fahren im öffentlichen Verkehr. Der Fokus dieser Testprojekte liegt mehrheitlich auf automatisierten Shuttlebussen (Barillère-Scholz et al. 2020: 18), die im Rahmen der Tests meist im Linienverkehr eingesetzt werden. Dabei zeigen sich jedoch bereits relevante Aspekte, die letztlich auch für den zukünftigen Einsatz der automatisierten Shuttlebusse oder anderer automatisierter Fahrzeugkonzepte im Rahmen flexibler bzw. alternativer Angebotsformen relevant sind. Im nächsten Abschnitt wird der Use Case des automatisierten Shuttlebusses daher noch näher beleuchtet und ein beispielhafter Überblick zu den verschiedenen Testprojekten gegeben.

Tabelle 3: Bedienungsformen von flexiblen und alternativen Angebotsformen mit Automatisierung

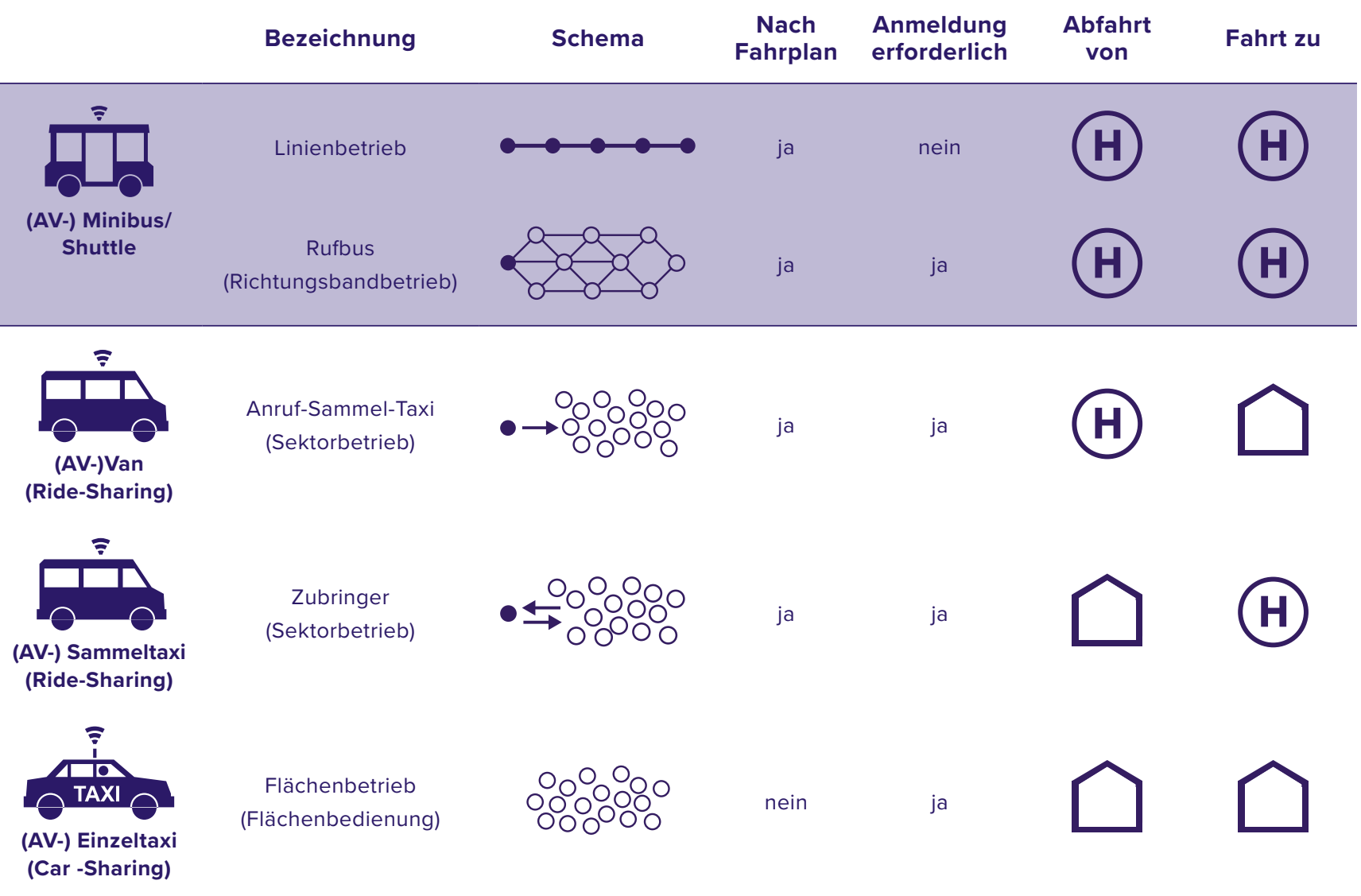

\begin{tabular}{|c|c|c|}
\hline$\bullet-$ & Haltestelle wird nach Fahrplan angefahren & Fahrt von/ \\
\hline$-\infty$ & Haltestelle wird bei Bedarf angefahren & \\
\hline O & $\begin{array}{l}\text { Bedienungsgebiet, innerhalb dessen überall } \\
\text { ein- oder ausgestiegen werden kann }\end{array}$ & $\begin{array}{c}\text { Fahrt von/zur } \\
\text { Haustür }\end{array}$ \\
\hline
\end{tabular}




\title{
3. AUTOMATISIERTE SHUTTLEBUSSE ALS VORRANGIGER USE CASE VON TESTPROJEKTEN IM ÖFFENTLICHEN VERKEHR
}

\begin{abstract}
Automatisierte Shuttlebusse bzw. fahrerlose, elektrische Kleinbusse stellen, wie in Abschnitt 2 dargestellt, einen speziellen Use Case automatisierter Fahrzeuge dar und stehen aktuell im Mittelpunkt der Testbemühungen öffentlicher Verkehrsunternehmen. Die aktuell auf dem Markt befindlichen und in den Testfeldern erprobten automatisierten Shuttlebusse wie beispielsweise NAVYA Arma oder EasyMile EZ10 entsprechen der Automatisierungsstufe 2 und fahren auf einer eigens genehmigten und vorbereiteten festen Route, auf der sie sowohl die Längs- als auch die Querführung übernehmen (vgl. Rentschler et al. 2020: 320). Während des Betriebs ist mehrheitlich noch ein/e Sicherheitsfahrerln bzw. Operatorln an Bord unterwegs. Zunehmend gibt es jedoch auch Testfahrten, bei denen das Fahrzeug nur über eine Leitzentrale überwacht wird.
\end{abstract}

Durch die geringe Kapazität der Shuttlebusse - meist sind sie auf 8 bis 12 Personen ausgelegt können die Modelle als sinnvolle und bedarfsgerechte Ergänzung des ÖV-Systems fungieren. Wie zuvor dargestellt, zeichnen sich grundsätzlich mehrere Anwendungsfelder eines automatisierten Shuttlebusses im ÖV ab, die jedoch aufgrund technologischer Einschränkungen bislang meist lediglich im Linienverkehr getestet werden (vgl. Derer/Geis 2020: 7; Földes/Csiszár 2018: 2).

Abbildung 2: Mögliche Einsatzfelder von automatisierten Shuttlebussen

Zubringerbus für Regionalverkehr Zwecke: Beruf-, Ausbildungs-, Freizeit- und Tourismusmobilität

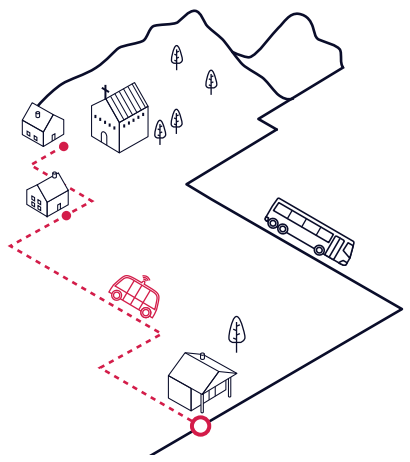

Dorfverbindungsbus

Zwecke: Berufs-, Freizeit- und Tourismusmobilit

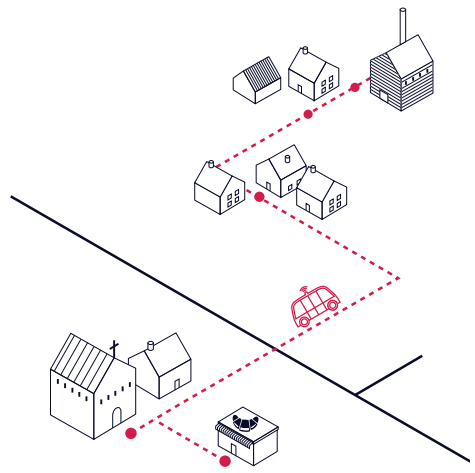

Erschließungsbus am Stadtrand

Zwecke: Freizeit- und Einkaufsmobilität

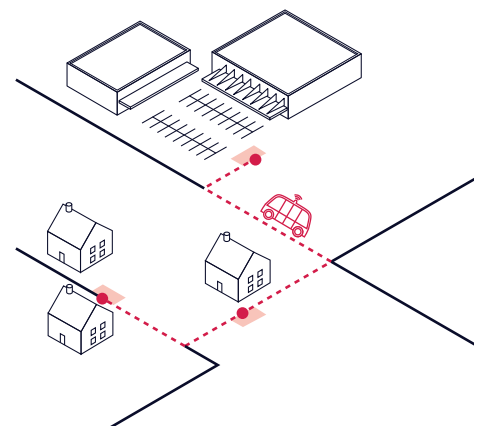

Nachbarschaftsbus in fragmentiertem Stadtraum mittlerer Dichte Zwecke: Berufs-, Ausbildungs- und Freizeitmobilität

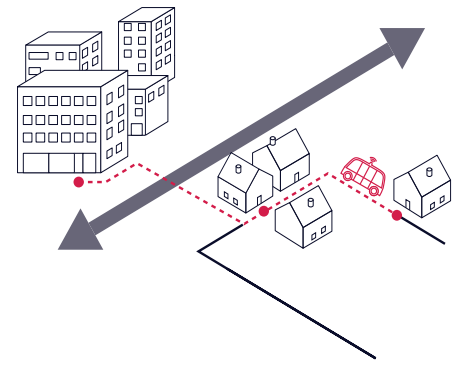

Campusbus

Zwecke: Berufs-, Ausbildungs- und

Gesundheitsmobilität

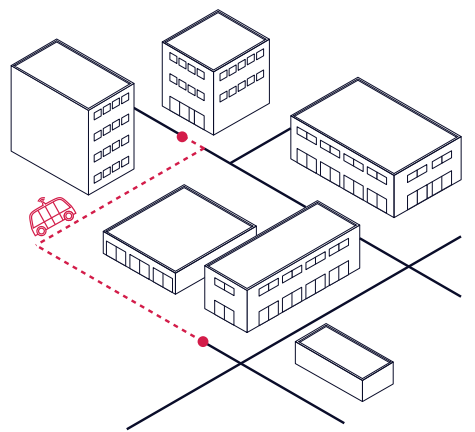


Zukünftig könnten automatisierte Shuttlebusse ein breites Einsatzfeld umfassen, flexibel eingesetzt werden und so bedarfsgerecht den klassischen Linienverkehr effizient ersetzen. Gerade im Bereich von geringer Verkehrsnachfrage, in weitläufigen Wohn- und Gewerbegebieten oder in großräumigen Krankenhauszentren sowie auf Universitäts- und Forschungscampus, könnten automatisierte Shuttlebusse dazu dienen, die Gebiete besser zu erschließen und anzubinden (vgl. Derer/Geis 2020: 7). Aufgrund des genannten Lückenschlusses im öffentlichen Verkehrsnetz tritt das Testen von automatisierten Shuttles auch immer häufiger in den Fokus von Kommunen, die bestrebt sind, einen „Transit-first“-Ansatz der Automatisierung zu fördern (vgl. Heinrichs et al. 2019: 248). Abbildung 2 gibt zusammenfassend einen Überblick zu möglichen Einsatzfeldern automatisierter Shuttlebusse.

In Europa wurden zwischen 2012 und 2016 die ersten Tests mit automatisierten Shuttlebussen auf öffentlichen Straßen im Rahmen des "CityMobil2“-Projekts (vgl. Alessandrini et al. 2015) durchgeführt. Davor wurden meist nur Demonstrationsprojekte vorgenommen. Seither wurden in Folge von Forschungsförderungen und der Anpassung rechtlicher Rahmenbedingungen zahlreiche weitere Testprojekte mit automatisierten Shuttlebussen in Europa eingerichtet. Abbildung 3 gibt einen Überblick zu Testprojekten mit automatisierten Shuttlebussen in Europa.

Abbildung 3: Standorte von Testprojekten und Demonstrationen mit automatisierten Shuttlebussen auf öffentlichen StraBen und Privatgeländen in Europa seit 2008 (kein Anspruch auf Vollständigkeit)

\begin{tabular}{|c|c|}
\hline Wien, $\mathrm{AT}^{+}$ & Frankfurt am Main, DE* \\
\hline Koppl, $\mathrm{AT}^{+}$ & Hamburg, DE \\
\hline Wiener Neustadt, AT & Aachen, $\mathrm{DE}^{\circ}$ \\
\hline Teesdorf, $\mathrm{AT}^{*}$ & Flughafen Weeze, DE \\
\hline Salzburg, AT* & Lahr, DE \\
\hline Pörtschach, AT & Neustadt /Weinstraße, DE \\
\hline Mechelen, BE* & Sylt, DE \\
\hline Spa-Francorchamps, BE* & Bad Birnbach, DE \\
\hline Han-sur-Lesse, BE & Wusterhausen/Dosse, DE \\
\hline Eigenbrakel, BE & Berlin, $\mathrm{DE}^{+}$ \\
\hline Marche-en-Famenne, BE* & Leipzig, DE* \\
\hline Brüssel, $\mathrm{BE}^{+}$ & Trikala, GR \\
\hline Flughafen Brüssel Zaventem, $\mathrm{BE}^{\circ}$ & Dublin, IR \\
\hline Aalborg Øst, DK & Oristano, IT \\
\hline Nordhavn, Kopenhagen, DK & Turin, IT \\
\hline Tallinn, $\mathrm{EE}^{+}$ & Luxembourg, LU \\
\hline Vantaa, FI & Contern, LU \\
\hline Helsinki, $\mathrm{Fl}^{+}$ & Svalbard, NO* \\
\hline Espoo, $\mathrm{Fl}^{+}$ & Gjesdal, NO \\
\hline Tempere, FI & Gjøvik, NO \\
\hline Kivikko, Helsinki, FI & Stavanger, NO \\
\hline Antibes, FR & Fornebu, NO \\
\hline La Rochelle, FR ${ }^{+}$ & Oslo, $\mathrm{NO}^{+}$ \\
\hline Sophia Antipolis, FR & Kongsberg, NO \\
\hline Civaux, FR & Trondheim, NO \\
\hline Lyon, FR FR $^{+}$ & Gdansk, PL \\
\hline Villeneuve-d'Ascq, FR & Castallón, SP* \\
\hline Rennes, $\mathrm{FR}^{+}$ & Donostia/San Sebastian, SI \\
\hline Paris, $\mathrm{FR}^{+}$ & Varuträsk, SE \\
\hline Versailles, $\mathrm{FR}^{+}$ & Stockholm, SE ${ }^{+}$ \\
\hline Sorigny, FR & Göteborg, SE ${ }^{+}$ \\
\hline Rouen, $\mathrm{FR}^{+}$ & Lausanne, $\mathrm{CH}$ \\
\hline Saclay, $\mathrm{FR}^{+}$ & Neuhausen, $\mathrm{CH}$ \\
\hline Boulogne-sur-Mer, FR & Genf, CH \\
\hline Toulouse, FR & Zug, CH \\
\hline Pibrac, FR & Sion, $\mathrm{CH}$ \\
\hline Verdun, FR & Bern, $\mathrm{CH}$ \\
\hline Reims, FR & Freiburg, $\mathrm{CH}$ \\
\hline Dünkirchen, FR & Cossonay, $\mathrm{CH}$ \\
\hline
\end{tabular}

Appelscha, NL Drimmelen, NL Noordwijk, NL Den Haag, NL Rotterdam, NL Scheemda, NL Flughafen Amsterdam Schiphol-Haarlem, NL* Wageningen, NL

Han-sur-Lesse, BE Wusterhausen/Dosse, DE Heathrow PRT, UK Eigenbrakel, BE

Marche-en-Famenne, BE Daventry, UK* Milton Keynes, UK Edinburgh, UK ${ }^{\circ}$ London, UK Manchester, UK ${ }^{+}$

\section{Aalborg Øst, DK}

Manchester, U

- Geplant für 2020

Mehrfache Testprojekte
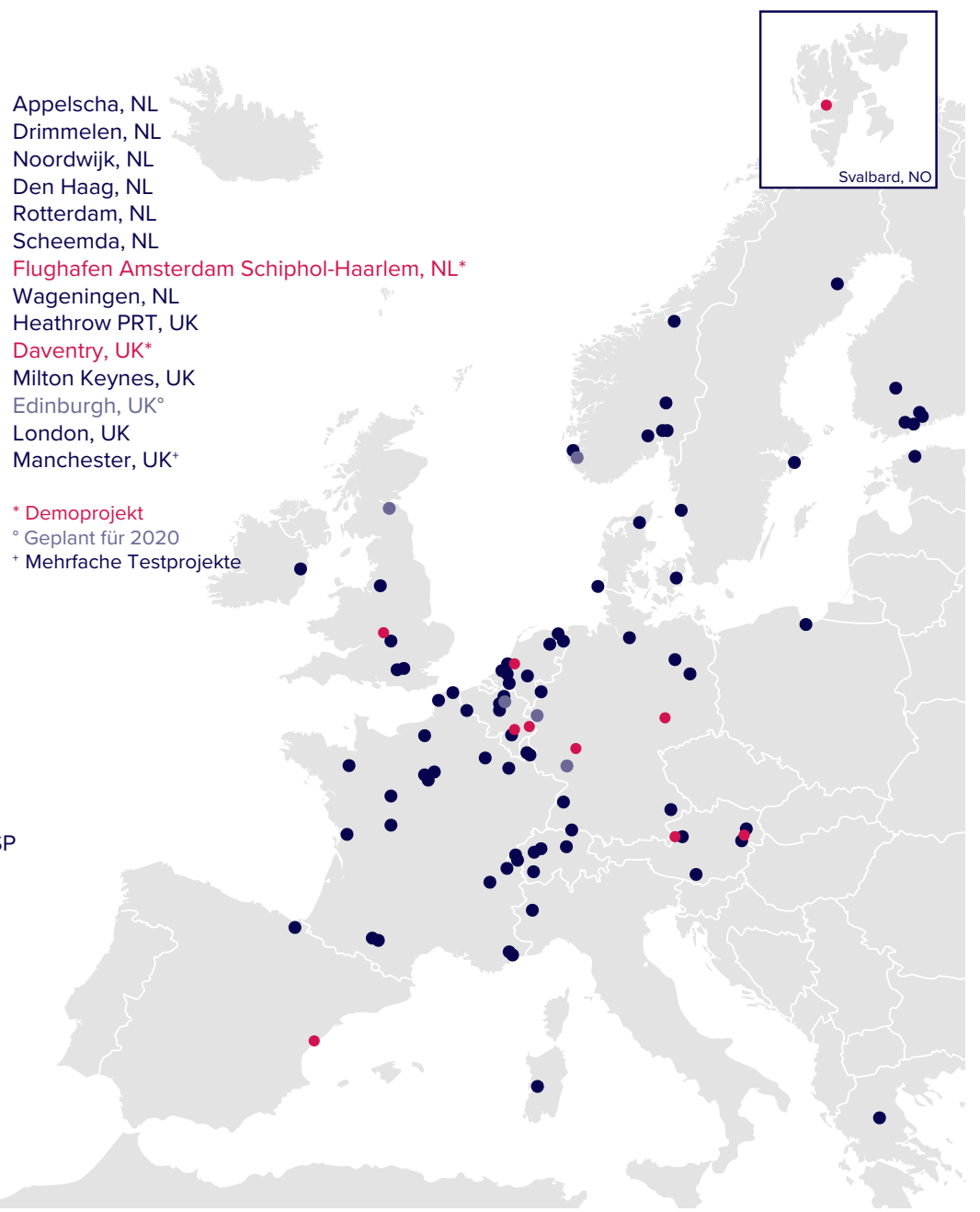

Quelle: eigene Darstellung nach Alessandrini (2016), Ainsalu et al. (2018) und Hagenzieker et al. (2020) 
In der Regel liegt der Fokus der bisherigen Testprojekte mit automatisierten Shuttlebussen auf folgenden Aspekten (vgl. Jürgens 2020):

- Technologische Machbarkeit: technische Aspekte, insbesondere Fahrzeugtechnologie, Infrastruktur (physisch und digital), Interaktion mit anderen Verkehrsteilnehmerlnnen und PassagierInnen (Mensch-Maschine-Interaktion), Verkehrssicherheit, Datensicherheit

- Organisatorische und betriebliche Machbarkeit: rechtliche und administrative Aspekte (z. B. Zulassungsverfahren zur Inbetriebnahme, Versicherung), betriebliche Aspekte/Service, Integration der automatisierten Shuttlebusse in das bestehende ÖV-System (MaaS, Schnittstellen, Dateninfrastruktur)

- Wirtschaftliche Machbarkeit: betriebswirtschaftliche Aspekte bzw. Finanzierung (z. B. Betriebskosten), Nutzerakzeptanz

- Soziale Dimension: Inklusion

Zwischen den einzelnen Testvorhaben zeigen sich jedoch unterschiedlich starke Forschungsschwerpunkte. Zudem lassen sich die Projekte auch in ihrer räumlichen Verortung sowie hinsichtlich ihrer verkehrlichen Integration und ihrer Betriebskonzepte unterscheiden.

Am Beispiel ausgewählter Testprojekte mit automatisierten Shuttlebussen in Europa werden im Folgenden die möglichen Schwerpunkte sowie auch die verschiedenen räumlichen Einsatzumgebungen und Betriebskonzepte, in denen automatisierte Shuttlebusse derzeit getestet werden, aufgezeigt. Um ein möglichst breites Spektrum abzudecken, wurden für die Gegenüberstellung Testprojekte in Wien und Koppl in Österreich, in Aalborg Øst in Dänemark, in Wusterhausen/Dosse in Deutschland und in Zug in der Schweiz ausgewählt (siehe auch Tab. 4 auf den folgenden Seiten).

Die Testprojekte ähneln sich allesamt hinsichtlich ihrer technologischen und formalen Kriterien. Jeweils im Linienbetrieb eingesetzt, verkehren die Shuttlebusse in der Regel auf Strecken zwischen 2 und 3,5 Kilometern und halten an bis zu 10 vordefinierten Haltestationen. In diesem Sinne erfüllen die Testbetriebe kaum die Erwartungen einer flexiblen Buchung oder Streckenführung, demonstrieren allerdings das langfristige Potential sowie die Grenzen eines ergänzenden Einsatzes im öffentlichen Verkehrsnetz. Um diese Aspekte zu bewerten, sind die getesteten Betriebsformen besonders im Kontext der räumlichen Gegebenheiten und lokalen Anforderungen zu betrachten. So wird im Zuge der Projekte meist die Anbindung der ersten bzw. letzten Meile mit automatisierten Shuttles getestet, die sich allerdings in ihrer räumlichen, topographischen und bedarfsgerechten Eignung unterscheiden:

- Im Projekt „auto.Bus - Seestadt“ in Wien kommen Shuttlebusse auf den Wohnstraßen eines dichten Neubauquartiers zum Einsatz, um dessen Anbindung an die U-Bahn-Endstation in Form eines Erschließungsbusses zu erproben. Hier erscheint hinsichtlich der hohen Bewohnerdichte weder die Fahrzeuggröße langfristig wirtschaftlich rentabel noch scheint die Konkurrenz zu aktiven Mobilitätsformen und neuen Formen der Mikromobilität zielführend.

- In Koppl, einem ländlichen Dorf in Österreich, wurde hingegen im Rahmen des Projekts „Digibus 2017“ die Anbindung der Ortsteile an eine regionale Busstrecke getestet, um in Zukunft Pendelverkehre in das überregionale Zentrum zu erleichtern (Zubringerbus für Regionalverkehr). Hierbei wird das Potential darin gesehen, zukünftig disperse Siedlungsteile bedarfsorientiert zu erschließen und dadurch für BewohnerInnen, Touristlnnen und den Warenverkehr eine leistbare Alternative zum privaten Automobil zu schaffen und dadurch den Standort zu stabilisieren. 
- Im Projekt „smartbusaalborg“ im dänischen Aalborg Øst wird der Lückenschluss im öffentlichen Verkehrsnetz durch den automatisierten Shuttle in Form eines Nachbarschaftsbusses erprobt, der auf einem nunmehr zentralen Fuß- und Radweg verkehrt, um unterschiedliche Wohngebiete und Einrichtungen eines hochfragmentierten Stadtteils miteinander zu verknüpfen. Hier steht nicht nur die tangentiale Verkehrsanbindung im Fokus, sondern vor allem die Mobilisierung der Bevölkerung und die soziale Integration eines bislang durch funktionalistische Planungsprinzipien geprägten suburbanen Bezirks von Aalborg.

- Auch in Wusterhausen/Dosse, einer Kleinstadt im Nordwesten von Brandenburg in Deutschland, liegt die Mobilisierung der Bewohnerlnnen und besonders der alternden Bevölkerung im Vordergrund des Testprojekts. Der automatisierte Shuttlebus erschließt die Kleinstadt, bindet sie an den regionalen Bahnverkehr an und soll in einer zweiten Phase auch entlegenere Ortsteile bedienen (Dorfverbindungsbus). Vor dem Hintergrund der Abwanderung, der hohen Auspendlerzahlen und der alternden Bevölkerung wird in der Erweiterung und Flexibilisierung des öffentlichen Nahverkehrs die Chance gesehen, die Region für Einheimische und TouristInnen zu attraktivieren sowie disperse Siedlungsgebiete als Wohnstandorte zu stabilisieren.

- Im Rahmen des Projekts „MyShuttle“ in Zug, einer Kleinstadt in der Schweiz, wurde der automatisierte Shuttlebus eingesetzt, um das im Norden der Stadt gelegene Areal des Unternehmens V-Zug besser an den Bahnhof anzubinden und so ein mögliches Angebot für PendlerInnen des Unternehmens V-Zug zu schaffen (Zubringerbus für Regionalverkehr). Speziell im Vordergrund stand hierbei die Integration des automatisierten Shuttles in die bestehenden Informationssysteme des öffentlichen Verkehrs.

Zusammenfassend gibt Tabelle 4 einen Überblick zu den betrachteten Testprojekten mit automatisierten Shuttlebussen.

Tabelle 4: Überblick zu ausgewählten Testprojekten mit automatisierten Shuttlebussen

\begin{tabular}{|c|c|c|c|c|c|}
\hline & Wien, AT & Aalborg Øst, DK & $\begin{array}{c}\text { Wusterhausen/ } \\
\text { Dosse, DE }\end{array}$ & Koppl, AT & Zug, CH \\
\hline Projekt & auto.Bus Seestadt & smartbusaalborg & autoNV OPR & Digibus $^{\oplus} 2017$ & MyShuttle \\
\hline $\begin{array}{l}\text { Dauer des } \\
\text { Testbetriebs }\end{array}$ & 06/2019-07/2020 & $03 / 2020-06 / 2021$ & $10 / 2019-06 / 2020$ & 04-11/2017 & 01/2019-12/2019 \\
\hline $\begin{array}{l}\text { Regionaler } \\
\text { Kontext bzw. } \\
\text { Gebietstyp }\end{array}$ & $\begin{array}{l}\text { - Stadtrand von } \\
\text { Wien bzw. Stadt- } \\
\text { randzentrum } \\
\text { - Stadtentwick- } \\
\text { lungsgebiet mit } \\
\text { hoher Dichte } \\
\text { und Nutzungs- } \\
\text { mischung und } \\
\text { Restriktionen für } \\
\text { private Fahr- } \\
\text { zeugnutzung } \\
\text { - positive Be- } \\
\text { völkerungsent- } \\
\text { wicklung }\end{array}$ & $\begin{array}{l}\text { - Suburbanes } \\
\text { Gebiet bzw. } \\
\text { Randgebiet der } \\
\text { städtischen Ag- } \\
\text { glomeration } \\
\text { - Stadtrandgebiet } \\
\text { aus den 1970er } \\
\text { Jahren mit städti- } \\
\text { schen Revitalisie- } \\
\text { rungsprojekten } \\
\text { - Positive } \\
\text { Bevölkerungs- } \\
\text { entwicklung }\end{array}$ & $\begin{array}{l}\text { - Freizeit- und } \\
\text { Tourismusort in } \\
\text { ländlicher Region } \\
\text { fernab städti- } \\
\text { scher Agglome- } \\
\text { ration } \\
\text { - Historisches } \\
\text { Dorfzentrum, dis- } \\
\text { perse Siedlungs- } \\
\text { struktur } \\
\text { - Überalterte und } \\
\text { schrumpfende } \\
\text { Bevölkerung } \\
\text { Freizeit- /Touris- } \\
\text { musverkehr }\end{array}$ & $\begin{array}{l}\text { - Ländliche Ge- } \\
\text { meinde östlich } \\
\text { von Salzburg }\end{array}$ & $\begin{array}{l}\text { - Kleinstadt südlich } \\
\text { von Zürich } \\
\text { - Historisches } \\
\text { Stadtzentrum } \\
\text { und dichte Sied- } \\
\text { lungsstruktur } \\
\text { - Positive } \\
\text { Bevölkerungs- } \\
\text { entwicklung } \\
\text { - Pendlerverkehr }\end{array}$ \\
\hline
\end{tabular}




\begin{tabular}{|c|c|c|c|c|c|}
\hline Projekt & auto.Bus Seestadt & smartbusaalborg & autoNV OPR & Digibus $^{\circledR} 2017$ & MyShuttle \\
\hline $\begin{array}{l}\text { Testumgebung } \\
\text { und Strecke }\end{array}$ & $\begin{array}{l}\text { Mischverkehr auf } \\
\text { Straßen in städ- } \\
\text { tischer Wohnge- } \\
\text { gend } \\
\text { ca. } 2 \text { km Länge, } \\
10 \text { Haltestellen }\end{array}$ & $\begin{array}{l}\text { Mischverkehr auf } \\
\text { einem Geh- und } \\
\text { Radweg } \\
\text { - } 2,1 \mathrm{~km} \text { Länge, } \\
10 \text { Haltestellen }\end{array}$ & $\begin{array}{l}\text { Mischverkehr auf } \\
\text { Dorfstraßen und } \\
\text { Landstraßen } \\
\text { - } 8 \mathrm{~km}, 18 \text { Halte- } \\
\text { stellen }\end{array}$ & $\begin{array}{l}\text { Mischverkehr auf } \\
\text { einer Landstraße } \\
\text { bzw. Dorfer- } \\
\text { schließungs- } \\
\text { straße } \\
\text { 1,4 km Länge, } \\
6 \text { Haltestellen }\end{array}$ & $\begin{array}{l}\text { - Mischverkehr auf } \\
\text { städtischer Orts- } \\
\text { straße } \\
\text { - } \begin{array}{l}1,5 \mathrm{~km} \text { Länge, } \\
3 \text { Haltestellen }\end{array}\end{array}$ \\
\hline $\begin{array}{l}\text { Verkehrliche } \\
\text { bzw. städte- } \\
\text { bauliche Integ- } \\
\text { ration bzw. Be- } \\
\text { triebskonzept }\end{array}$ & $\begin{array}{l}\text { Verknüpfung } \\
\text { der U-Bahn-End- } \\
\text { haltestelle mit } \\
\text { benachbarten } \\
\text { dichten Wohnge- } \\
\text { bieten }\end{array}$ & $\begin{array}{l}\text { Verknüpfung } \\
\text { eines neuen } \\
\text { Stadtteilzentrums } \\
\text { mit sozialen Ein- } \\
\text { richtungen, frag- } \\
\text { mentierenden } \\
\text { Wohngebieten } \\
\text { sowie einem Uni- } \\
\text { versitätscampus }\end{array}$ & $\begin{array}{l}\text { Verknüpfung } \\
\text { des Stadt- bzw. } \\
\text { Ortszentrums mit } \\
\text { dem Bahnhof, } \\
\text { dem Shopping- } \\
\text { zentrum sowie } \\
\text { dem Super- } \\
\text { markt und einem } \\
\text { Wohngebiet am } \\
\text { Stadtrand }\end{array}$ & $\begin{array}{l}\text { Verknüpfung des } \\
\text { Dorfzentrums mit } \\
\text { einem regionalen } \\
\text { (ÖV)-Verkehrs- } \\
\text { korridor }\end{array}$ & $\begin{array}{l}\text { Verknüpfung des } \\
\text { Bahnhofs bzw. } \\
\text { Einkaufszent- } \\
\text { rums Metalli mit } \\
\text { dem Areal des } \\
\text { Unternehmens V- } \\
\text { Zug (Bedienung } \\
\text { erste und letzte } \\
\text { Meile) }\end{array}$ \\
\hline $\begin{array}{l}\text { Kommunale } \\
\text { Beteiligung }\end{array}$ & $\begin{array}{l}\text { Verankert bei } \\
\text { den öffentlichen } \\
\text { Verkehrsbe- } \\
\text { trieben Wiener } \\
\text { Linien } \\
\text { - Städtische Ver- } \\
\text { waltung in Ab- } \\
\text { stimmungspro- } \\
\text { zessen involviert }\end{array}$ & $\begin{array}{l}\text { Verankert in der } \\
\text { Stadt und Land- } \\
\text { schaftsverwal- } \\
\text { tung der Stadt } \\
\text { Aalborg } \\
\text { Breite Be- } \\
\text { teiligung der } \\
\text { städtischen Ver- } \\
\text { waltung }\end{array}$ & $\begin{array}{l}\text { - Landkreis Ost- } \\
\text { prignitz-Ruppin } \\
\text { und } \\
\text { - Betriebe vom } \\
\text { öffentlichen } \\
\text { Verkehrsbetrieb } \\
\text { ORP-Busse } \\
\text { als assoziierte } \\
\text { Partner }\end{array}$ & $\begin{array}{l}\text { Bereitstellung } \\
\text { der Rahmenbe- } \\
\text { dingungen durch } \\
\text { die Gemeinde } \\
\text { Koppl }\end{array}$ & $\begin{array}{l}\text { - Stadt Zug als } \\
\text { Partner im } \\
\text { Projekt }\end{array}$ \\
\hline
\end{tabular}

\section{Vorrangige Themen im Projekt}

\begin{tabular}{|c|c|c|c|c|c|}
\hline Projekt & auto.Bus Seestadt & smartbusaalborg & autoNV OPR & Digibus $^{\circledR} 2017$ & MyShuttle \\
\hline \multicolumn{6}{|c|}{ Technologische Machbarkeit } \\
\hline $\begin{array}{l}\text { Fahrzeug- } \\
\text { technologie }\end{array}$ & $\checkmark$ & $\checkmark$ & $\checkmark$ & $\checkmark$ & $\checkmark$ \\
\hline $\begin{array}{l}\text { Infrastruktur } \\
\text { (physisch und } \\
\text { digital) }\end{array}$ & $\checkmark$ & $\checkmark$ & $\checkmark$ & $\checkmark$ & $\checkmark$ \\
\hline \multicolumn{6}{|l|}{$\begin{array}{l}\text { Interaktion } \\
\text { mit anderen } \\
\text { Verkehrsteilneh- } \\
\text { merlnnen (und } \\
\text { PassagierInnen) }\end{array}$} \\
\hline $\begin{array}{l}\text { Verkehrs- } \\
\text { sicherheit }\end{array}$ & $\checkmark$ & & & $\checkmark$ & \\
\hline $\begin{array}{l}\text { Daten- } \\
\text { sicherheit }\end{array}$ & $\checkmark$ & $\checkmark$ & & $\checkmark$ & \\
\hline \multicolumn{6}{|c|}{ Organisatorische und betriebliche Machbarkeit } \\
\hline $\begin{array}{l}\text { Rechtliche und } \\
\text { administrative } \\
\text { Aspekte }\end{array}$ & & & $\checkmark$ & $\checkmark$ & $\checkmark$ \\
\hline
\end{tabular}




\begin{tabular}{|c|c|c|c|c|c|}
\hline Projekt & auto.Bus Seestadt & smartbusaalborg & autoNV OPR & Digibus $^{\oplus} 2017$ & MyShuttle \\
\hline $\begin{array}{l}\text { Betriebliche } \\
\text { Aspekte }\end{array}$ & $\checkmark$ & $\checkmark$ & $\checkmark$ & & $\checkmark$ \\
\hline $\begin{array}{l}\text { Integration in } \\
\text { das bestehende } \\
\text { ÖV-System }\end{array}$ & $\checkmark$ & $\checkmark$ & $\checkmark$ & $\checkmark$ & $\checkmark$ \\
\hline \multicolumn{6}{|c|}{ Wirtschaftliche Machbarkeit } \\
\hline $\begin{array}{l}\text { Betriebswirt- } \\
\text { schaftliche } \\
\text { Aspekte/ } \\
\text { Finanzierung }\end{array}$ & & & $\checkmark$ & & $\checkmark$ \\
\hline $\begin{array}{l}\text { Nutzer- } \\
\text { akzeptanz }\end{array}$ & $\checkmark$ & & $\checkmark$ & $\checkmark$ & $\checkmark$ \\
\hline \multicolumn{6}{|c|}{ Soziale Dimension } \\
\hline Soziale Inklusion & & $\checkmark$ & & & \\
\hline
\end{tabular}

Quelle: eigene Darstellung

Um spezifische Themen der ausgewählten Testprojekte mit automatisierten Shuttlebussen noch detaillierter zu beleuchten, stellen die folgenden beiden Abschnitte jeweils Vertiefungen zu zwei der beschriebenen Tests dar und geben einen Einblick in unterschiedliche, jedoch beidseits relevante Aspekte beim Testen automatisierter Shuttlebusse und deren Betrieb. In der ersten Vertiefung wird am Beispiel des Projekts „autoNV OPR“ in Ostprignitz-Ruppin, Deutschland, ein Einblick in die technischen und rechtlichen Aspekte des Einsatzes automatisierter Shuttlebusse im ÖV gegeben. Die zweite Vertiefung gibt am Beispiel des Projekts "MyShuttle“ in Zug, Schweiz, einen Einblick in den Betrieb und die Integration automatisierter Shuttles in den bestehenden öffentlichen Verkehr.

\title{
4. TECHNISCHE UND RECHTLICHE ASPEKTE BEIM TESTEN AUTOMATISIERTER SHUTTLEBUSSE IM ÖFFENTLICHEN VERKEHR
}

\author{
am Beispiel des Projekts „autoNV OPR“ in Ostprignitz-Ruppin, Deutschland \\ Arne Holst, Alexander Egoldt, Thomas Richter
}

Das Verbundprojekt „autoNV OPR“ erforscht im ländlichen Raum, konkret im Landkreis Ostprignitz-Ruppin im Nordwesten von Brandenburg in Deutschland, den Einsatz und die Auswirkungen automatisierter Kleinbusse im öffentlichen Straßenraum. Das Projektkonsortium besteht aus den Universitäten TU Dresden und TU Berlin, der Regionalentwicklungsgesellschaft Nordwestbrandenburg, der Ostprignitz-Ruppiner Personennahverkehrsgesellschaft und den Unterauftragnehmern IGES Institut und Büro autoBus. Im Rahmen des Projekts werden die Rahmenbedingungen für den verkehrlichen Einsatz von automatisiert fahrenden Betriebsformen sowie die Akzeptanz der NutzerInnen und StakeholderInnen untersucht. Zusätzlich werden Szenarien und Auswirkungen automatisierter Betriebsformen bezogen auf die Finanzierungsroutine des öffentlichen Verkehrs erforscht und Aussagen zur Übertragbarkeit hergeleitet. Der Projektstart erfolgte im Herbst 2017 und die im Projekt entstandene automatisierte Shuttlebuslinie ist seit Juli 2019 im Betrieb. Im Folgenden werden technische und rechtliche Aspekte beim Betrieb des automatisierten Shuttlebusses näher beleuchtet. 


\subsection{DER VERWENDETE SHUTTLEBUS}

Im Projekt wird das Shuttle EZ 10 der zweiten Generation des französischen Herstellers EasyMile verwendet (Abb. 4). Das Shuttle wird vom Hersteller als Fahrzeug der Automatisierungsstufe 4 eingestuft - entspricht aber, wie eingangs beschrieben, jedoch eigentlich nur der Automatisierungsstufe 2. Die Maße des Shuttles belaufen sich auf 4,02 × 2,00 x 2,87 Meter $(L \times B \times H)$. Es bietet sechs Sitzplätze, ist elektrisch angetrieben und kann manuell ausschließlich mittels Fernbedienung gesteuert werden. Das Shuttle hat eine technisch maximale Höchstgeschwindigkeit von $45 \mathrm{~km} / \mathrm{h}$, wobei es für das Projekt bis $20 \mathrm{~km} / \mathrm{h}$ zugelassen ist. Im Betrieb weist es jedoch eine Höchstgeschwindigkeit von 15 km/h auf (vgl. EasyMile 2019a). Mittels GPS, Korrektursignal, Trägheitssensoren, Odometrie und einer vorgespeicherten Karte kann sich der Shuttlebus lokalisieren und die vorgegebene Trajektorie abfahren (vgl. EasyMile 2019b). Die Karte, samt Trajektorie und Streckenumgebung, wird vor dem Betrieb während zahlreicher Testfahrten erstellt. Hierzu scannt der Shuttlebus seine Streckenumgebung, während er im manuellen Modus betrieben wird (vgl. Rutanen/Arffman 2017). Der Shuttlebus nutzt auf dem Dach zur Lokalisation montierte Lidarsensoren und hinterlegt in der Karte alle gescannten geometrischen Merkmale in entsprechender Höhe (vgl. Regional Transportation District 2019). Durch den Abgleich der gespeicherten geometrischen Merkmale und der in Echtzeit detektierten Daten kann sich der Shuttlebus durch Wiedererkennung der Merkmale selbst verorten. Diese Merkmale werden folgend als Landmarker bezeichnet. Hindernisse auf einer Höhe von 35 Zentimetern erkennt das Fahrzeug mittels seiner vier Sicherheits-Lidarsensoren, die eine 360-Grad-Hindernisdetektion ermöglichen (vgl. ebd.).

Abbildung 4: Das verwendete Shuttle EZ 10 im Betrieb

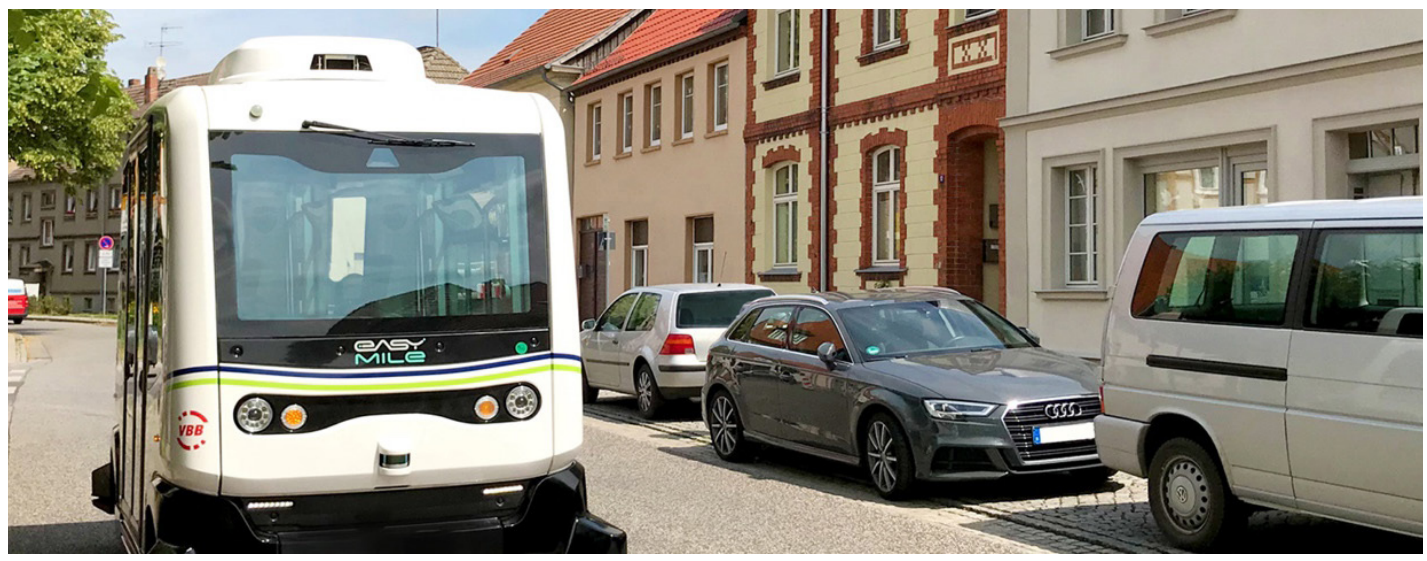

Foto: www.autonv.de

\subsection{STRECKENFINDUNG, INFRASTRUKTURANPASSUNG UND ORIENTIERUNG DES SHUTTLEBUSSES}

Hinsichtlich des Einsatzgebietes zum Testen des automatisierten Shuttlebusses fand durch die Festsetzung des Landkreises Ostprignitz-Ruppin als Testgebiet in der Projektskizze bereits eine erste Abgrenzung statt. Für eine weitere Einschränkung möglicher Einsatzgebiete wurden im Anschluss alle Knotenpunkte des übergeordneten Verkehrs (PlusBus und Bahnverkehr) im Landkreis erfasst, damit für die automatisierte Buslinie entsprechende Anbindungen vorhanden sind. Hierdurch wird die Buslinie Teil des ÖPNV-Netzes in Ostprignitz-Ruppin und kann durch ihre Integration einen Betrag zur Sicherung der Daseinsvorsorge leisten. 
Aufgrund der technischen Voraussetzungen kommt für den Betrieb des Busses bisher nur die letzte Meile in Frage. Hierfür wurde im nächsten Schritt der Bedarf anhand der Einwohnerzahlen der Ortschaften und Strukturdaten evaluiert. Durch diese zwei Schritte wurden im gesamten Landkreis Ostprignitz-Ruppin 25 potentielle Strecken sondiert, die im Anschluss auf weitere Kriterien geprüft wurden. Hierzu zählten unter anderem die Verkehrsdichte, Streckenlänge, Straßenkategorie, Höchstgeschwindigkeit, Mobilfunknetz und Anpassungsbedarf. Durch dieses Verfahren wurden zwei Strecken identifiziert und letztlich die Strecke in der Gemeinde Wusterhausen/Dosse ausgewählt.

Die ausgewählte Strecke für den Betrieb der automatisierten Buslinie ist insgesamt 8 Kilometer lang und verbindet den dezentral östlich liegenden Bahnhof der Gemeinde Wusterhausen/Dosse, der auch eine Anbindung zum PlusBus (örtliches ÖV-Angebot) besitzt, mit dem Stadtzentrum, den dortigen Supermärkten, dem Pflegeheim und einer dezentralen Wohnsiedlung sowie mit der Seenlandschaft am nördlichen Stadtrand. Unter Berücksichtigung der technischen Fähigkeiten des Fahrzeugs wurde die Einführung der Strecke in drei Stufen bzw. Abschnitte (gelb, rot, grün) realisiert. Die Streckenlänge kann aufgrund dieser Stufen bzw. Streckenabschnitte 2, 4 und 8 Kilometer betragen (Abb. 5).

Abbildung 5: Überblick der Strecke des automatisierten Shuttlebusses in der Gemeinde Wusterhausen/Dosse

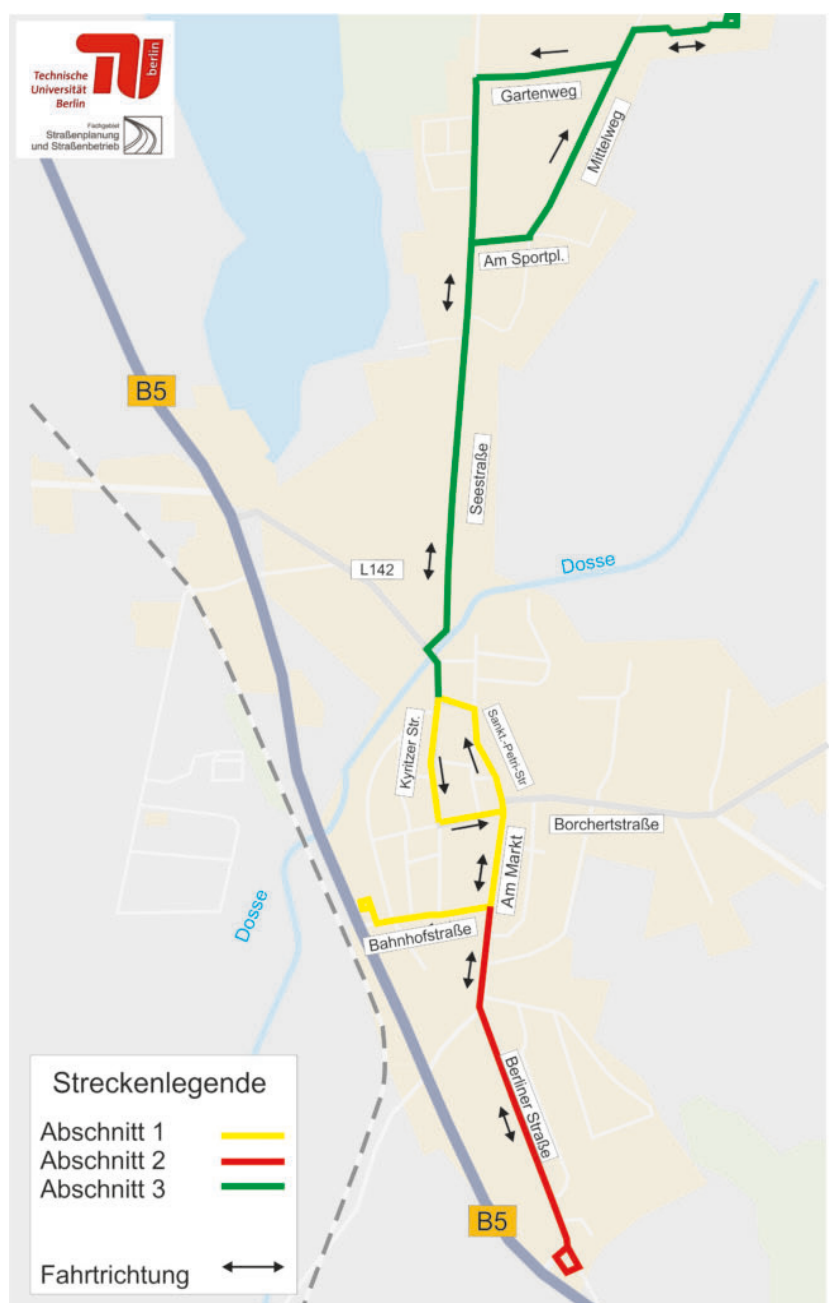

Quelle: www.autonv.de/fahrplan-strecke 
Zur Vorbereitung des jeweiligen Abschnitts gibt der Fahrzeughersteller (EasyMile) eine Bewertung ab - samt Empfehlungen zu infrastrukturellen Anpassungen. Am Beispiel des zweiten Streckenabschnitts (roter Abschnitt in Abb. 5) erfolgt im Folgenden eine Beschreibung solcher Anpassungen hinsichtlich Infrastruktur und Orientierung des Shuttlebusses.

Der zweite Streckenabschnitt, die Berliner Straße, ist gemäß der Forschungsgesellschaft für Straßen- und Verkehrswesen e. V. (FGSV 2006) eine örtliche Einfahrtstraße in der Stadt Wusterhausen und hat eine durchschnittliche tägliche Verkehrsstärke von $646 \mathrm{Kfz}$ pro 24 Stunden (Verkehrszählung 2018) sowie viele Grundstückszufahrten. Die Straße weist eine Fahrbahnbreite von 8,7 Metern auf, das Parken ist an beiden Fahrbahnrändern grundsätzlich erlaubt. Die zulässige Höchstgeschwindigkeit liegt bei 50 km/h (Abb. 6, links: „Ausgangslage“). Da die Trajektorie des Shuttlebusses nicht dynamisch änderbar ist, muss gewährleistet sein, dass sie nicht durch parkende Fahrzeuge blockiert wird. Eine Verlegung der Trajektorie in die Fahrbahnmitte ist jedoch aufgrund des deutschen Rechtsfahrgebotes ausgeschlossen. Um dieses Gebot nicht zu missachten, wurden während des Betriebs ein einseitiges Halteverbot auf der westlichen Straßenseite eingeführt und auf der östlichen Straßenseite Parkstände in Längsaufstellung markiert. Somit kann die Trajektorie Richtung Süden am Fahrbahnrand verlaufen und in Richtung Norden entlang des Parkstreifens. Hierdurch werden Konflikte zweier entgegenkommender Fahrzeuge im Straßenraum der Berliner Straße verhindert, da der Shuttlebus nicht mehr antizipieren muss, wie sich das entgegenkommende Fahrzeug verhalten wird. Zusätzlich wurde die maximale Höchstgeschwindigkeit aufgrund der schwierigen Fahrbahnoberflächenbeschaffenheit, mitunter verursacht durch Fahrbahnschäden und Kopfsteinpflaster, auf $30 \mathrm{~km} / \mathrm{h}$ heruntergesetzt. Die Planung des Halteverbotes und der Parkflächenmarkierung (rote Linie) ist der Mitte der Abbildung 6 und die Situation nach der Ausführung der rechten Seite der Abbildung 6 zu entnehmen.

Abbildung 6: Überblick der Infrastrukturanpassung für den Streckenabschnitt 2, Berliner Straße in Wusterhausen/Dosse, in den drei Phasen Ausgangslage, Planung und Ausführung

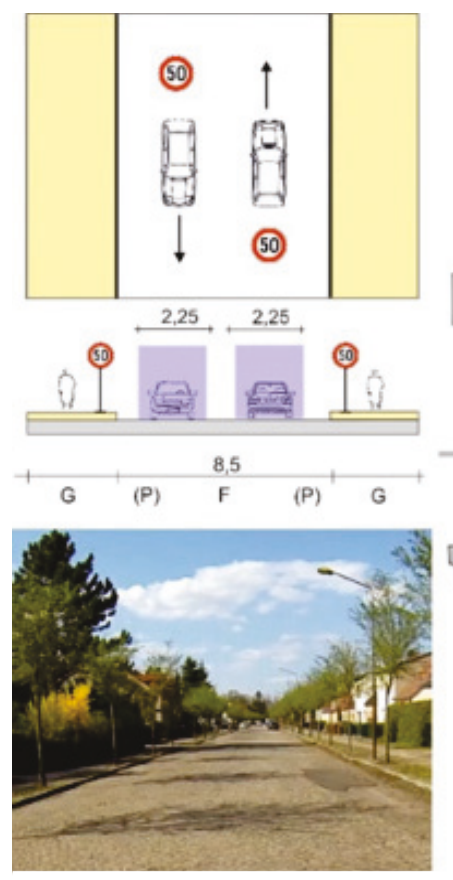

Ausgangslage
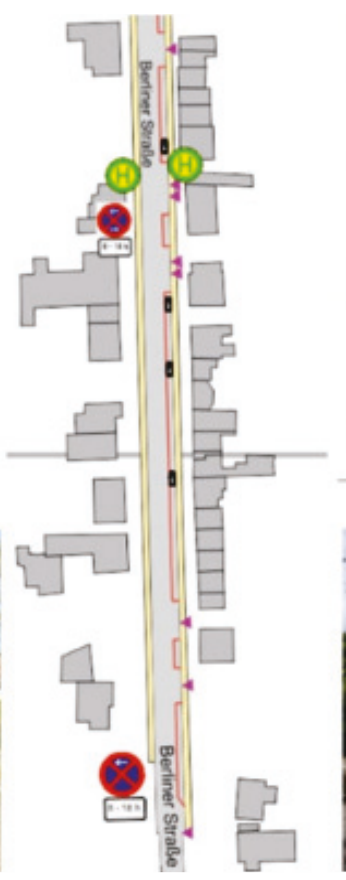

Planung
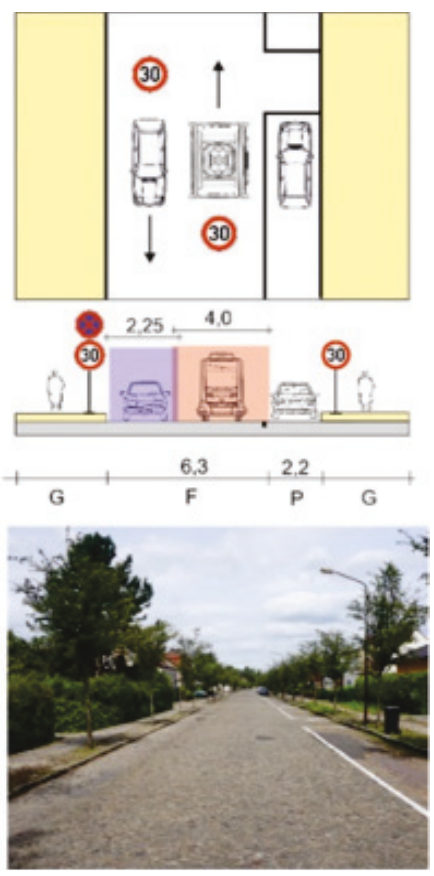

Ausführung

Quelle: eigene Darstellung; Foto links vom 17.4.2018, Foto rechts vom 4.7.2019; Fotos: Holst/Egoldt 
Die Querschnittsdarstellungen in Abbildung 6 zeigen die Sicherheitsräume gemäß FGSV (2006) und die des Shuttles (vgl. Rutanen/Arffman 2017). Im rechten Querschnitt ist zu erkennen, dass sich die Sicherheitsräume aufgrund der Maßnahmen schneiden, was wiederum zu einer Reduzierung der Geschwindigkeit des Shuttlebusses an einem solchen Punkt führt. Die Einführung eines beidseitigen Halteverbotes war aufgrund des hohen Stellplatzbedarfs nicht realisierbar.

Für die Orientierung des Shuttlebusses sind aufgrund des Lokalisationsverfahrens mittels Wiedererkennung von Landmarkern statische, geometrische Merkmale im Umfeld der Strecke essentiell. Durch die Positionierung des Lokalisations-Lidarsensors auf dem Dach des Shuttlebusses und des geringen Öffnungswinkels des Sensors wurden geometrische Objekte in einer Höhe von ca. 3 Metern benötigt (vgl. EasyMile 2019b). Hierfür sind Gebäude bzw. Gebäudeecken nutzbar, welche auf innerörtlichen Straßen zum Großteil vorhanden sind. Im südlichen Teil der Berliner Straße existierte jedoch ein anbaufreies Gebiet, in dem keine Landmarker vorhanden waren. Nach Absprache mit dem Hersteller EasyMile wurden in diesem Gebiet daher drei künstliche Landmarker in Form von Schildern aufgestellt. Um möglichst wenig in den StraBenraum einzugreifen, wurden diese Schilder an vorhandenen Laternenmasten angebracht. Die Ausgangslage, Planung mit Maßen und Ausführung ist der Abbildung $7 \mathrm{zu}$ entnehmen. Auch im dritten Streckenabschnitt existierten anbaufreie Straßenzüge. Hier konnten jedoch Bäume am Fahrbahnrand als Landmarker verwendet werden, da sie die entsprechenden Anforderungen erfüllten.

Abbildung 7: Überblick zur Umfeldanpassung für die Orientierung des Shuttles im Streckenabschnitt 2, Berliner Straße in Wusterhausen/Dosse, in den drei Phasen Ausgangslage, Planung und Ausführung

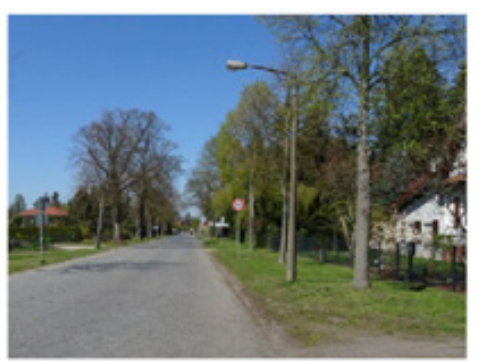

Ausgangslage

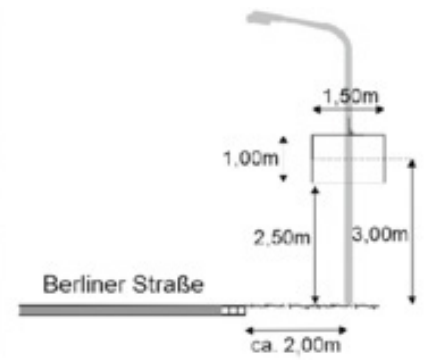

Planung

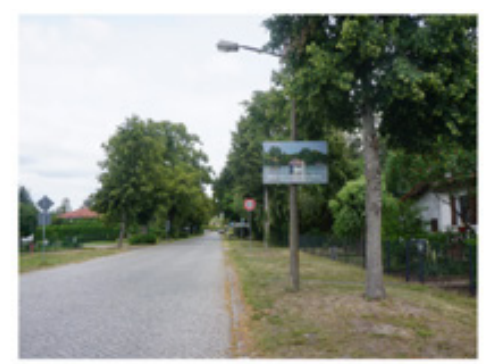

Ausführung

Quelle: eigene Darstellung; Foto links vom 17.4.2018, Foto rechts vom 4.7.2019; Fotos: Holst/Egoldt

\subsection{ZULASSUNG UND INBETRIEBNAHME}

Für den Betrieb des automatisierten Shuttlebusses war, wie für jedes auf öffentlicher Straße genutzte Fahrzeug, eine Zulassung erforderlich. Im Rahmen des Projekts erfolgte die Zulassung mittels einer Ausnahmegenehmigung. Für das Erhalten einer solchen Ausnahmegenehmigung ist in Deutschland grundsätzlich ein Gutachten einer amtlichen Prüfstelle erforderlich. Da in diesem Gutachten auch die Strecke sowie der Zeitraum des Projekts enthalten sein muss, ist sowohl die Ausnahmegenehmigung als auch die Zulassung des Shuttlebusses strecken- und zeitraumgebunden. Nach der erfolgreichen Zulassung wurde die Linienkonzessionierung beantragt. Hierfür mussten zahlreiche Unterlagen wie die Linienführung der Buslinie samt den Positionen und Namen aller Haltestellen sowie die Fahrzeugzulassung eingereicht werden. Erst mit erfolgreicher Linienkonzession war die Inbetriebnahme der Linie gemäß in Deutschland geltendem Recht möglich. Zusätzlich bedurfte es jedoch auch noch der Genehmigung des Fahrzeugherstellers, der erst nach Erfüllung der zuvor von ihm angegebenen infrastrukturellen Änderungsmaßnahmen sein Einverständnis für den Betrieb gab. 
Im Rahmen des Betriebs des Shuttlebusses erfolgten in den ersten Tagen der Freigabe eines Abschnitts Probefahrten ohne Fahrgäste, damit sich alle Verkehrsteilnehmerlnnen und insbesondere die Begleitpersonen an die Fahreigenschaften des Shuttlebusses gewöhnen konnten. Die Begleitpersonen sind nicht nur aufgrund rechtlicher Bestimmungen notwendig, sie müssen auch Fahraufgaben erfüllen. Die Fahrt muss an Knotenpunkten, damit der Shuttlebus sie passieren kann, durch die Begleitperson aktiv freigegeben werden. Aufgrund des Sicherheitsraums des Shuttlebusses kommt es auf Straßen mit Regelquerschnitten gemäß der RASt 06 (vgl. FGSV 2006) bei Gegenverkehr immer zu Bremsvorgängen, da sich das entgegenkommende Fahrzeug im Sicherheitsraum des Shuttlebusses befindet. Auch ein dynamischer Überholvorgang anderer Verkehrsteilnehmerlnnen, gefolgt von einem engen Einscheren vor dem Shuttlebus, führt zu Verzögerungen. Diese Verzögerungen haben Auswirkungen auf den Betrieb des Shuttlebusses und wurden von den Fahrgästen als negativ empfunden.

\title{
4.4 BISHERIGE ERKENNTNISSE
}

Die aufgeführten Erfahrungen spiegeln das Zulassungsverfahren, die Streckenfindung sowie deren Anpassung und eine Einschätzung des Betriebs nach einer ca. sechsmonatigen Betriebsdauer wider. Die Wahl des Herstellers ist für den Ablauf des Projektes bedeutend: Er nimmt sowohl bei der Streckenwahl als auch bei der Vorgabe infrastruktureller Anpassungen eine tragende Rolle ein. Auch die Bedarfe der NutzerInnen sind von großer Bedeutung. Bereits während der Freigabe des ersten Abschnitts wird der Wunsch nach einer Verlängerung der Strecke, insbesondere von der älteren Bevölkerung, geäußert. Jedoch zeigt sich auch, dass sich ein Ortsverkehr in einer Kleinstadt vor allem zeitlich an die Bedarfe der Fahrgäste richten muss: Zum einen ist eine Anpassung der Geschwindigkeit sowie eine Erhöhung der Zuverlässigkeit nötig, zum anderen muss auch der Fahrplan mit den Bedarfen der Nutzerlnnen abgestimmt werden. Das Erstellen eines bedarfsgerechten Fahrplans macht es umso schwerer, eine traditionelle Buslinie für eine Kleinstadt im ländlichen Raum zu etablieren und fordert das Prüfen von Alternativen. Zusätzlich sind derzeit infrastrukturelle Maßnahmen für einen möglichst störungsfreien Betrieb notwendig, die mit der Weiterentwicklung automatisierter Fahrfunktionen obsolet werden könnten und daher vermieden werden sollten.

\section{BETRIEB UND INTEGRATION AUTOMATISIERTER SHUTTLEBUSSE IN DEN ÖFFENTLICHEN VERKEHR}

\author{
am Beispiel des Projekts „MyShuttle“ in Zug, Schweiz \\ Zoltán László
}

Im Rahmen des Projekts „MyShuttle“ wurden unter Nutzung eines automatisierten Shuttlebusses verschiedene Einsatzmöglichkeiten von automatisierten Fahrzeugen und darauf basierende Angebotskonzepte analysiert, entwickelt und in einem ausgedehnten Feldtest in der Stadt Zug (etwa 30000 Einwohner) in der Schweiz getestet. Ausgangspunkt des Projekts waren die durch das automatisierte Fahren angestoßenen, radikalen Veränderungen im Personenverkehr. Insbesondere die mit dem automatisierten Fahren verbundenen möglichen Chancen für ein kundenfreundlicheres, effizienteres, umweltfreundlicheres und kostengünstigeres Gesamtverkehrssystem - im Sinne einer Konvergenz des öffentlichen und des individuellen Verkehrs (ÖIV) mit dem Fokus auf geteilte Fahrten (Sharing) und Mitfahrservices - bewegten die Schweizer Bundesbahnen (SBB) dazu, sich auf diese Veränderungen vorzubereiten, um den Kundlnnen auch zukünftig ein entsprechendes Angebot bieten zu können. 
Aus diesen Überlegungen heraus wurde das Projekt "MyShuttle“ durch die SBB, als größtes Personenbeförderungsunternehmen der Schweiz, gemeinsam mit dem Schweizer Unternehmen Mobility Carsharing, den Zugerland Verkehrsbetrieben, dem Standortpartner Stadt Zug sowie dem Technologiecluster Zug, gestartet. Der Fokus lag dabei in der betrieblichen Erprobung auf den drei Schwerpunkten: (1) Integrierbarkeit von automatisierten Fahrzeugen in das bestehende Kundeninformationssystem des öffentlichen Verkehrs, (2) konkrete Erfahrungen zur technischen Maturität der verfügbaren Soft- und Hardware und (3) die Kundenakzeptanz gegenüber automatisierten Shuttleservices. Im Januar 2019, nach eineinhalb Jahren Planung und Vorbereitung, startete der einjährige Pilotbetrieb. „MyShuttle“ war damit der erste automatisierte Shuttlebus in der Schweiz, der auf einer durchgehend hochfrequentierten öffentlichen Straße im Mischverkehr betrieben wurde. Im Folgenden werden der Betrieb und die Integration des automatisierten Shuttlebusses in den bestehenden öffentlichen Verkehr näher beleuchtet.

\subsection{VERWENDETER SHUTTLEBUS UND BETRIEB}

Bis zum Beginn des Projektvorhabens hatte keiner der führenden Technologieanbieter bzw. Fahrzeughersteller im Gebiet automatisierter Fahrzeuge eingewilligt, Fahrzeuge in der Schweiz zu testen oder in Pilotprojekte partnerschaftlich einzubringen. Im Rahmen des „MyShuttle“-Projekts gingen die Verantwortlichen daher pragmatisch vor und beschafften sich den aus Projektsicht passendsten und erwerbbaren automatisierten Shuttlebus: das Modell EZ1O vom französischen Start-up EasyMile (Abb. 8).

Abbildung 8: Das verwendete Shuttle EZ 10 im Betrieb in Zug

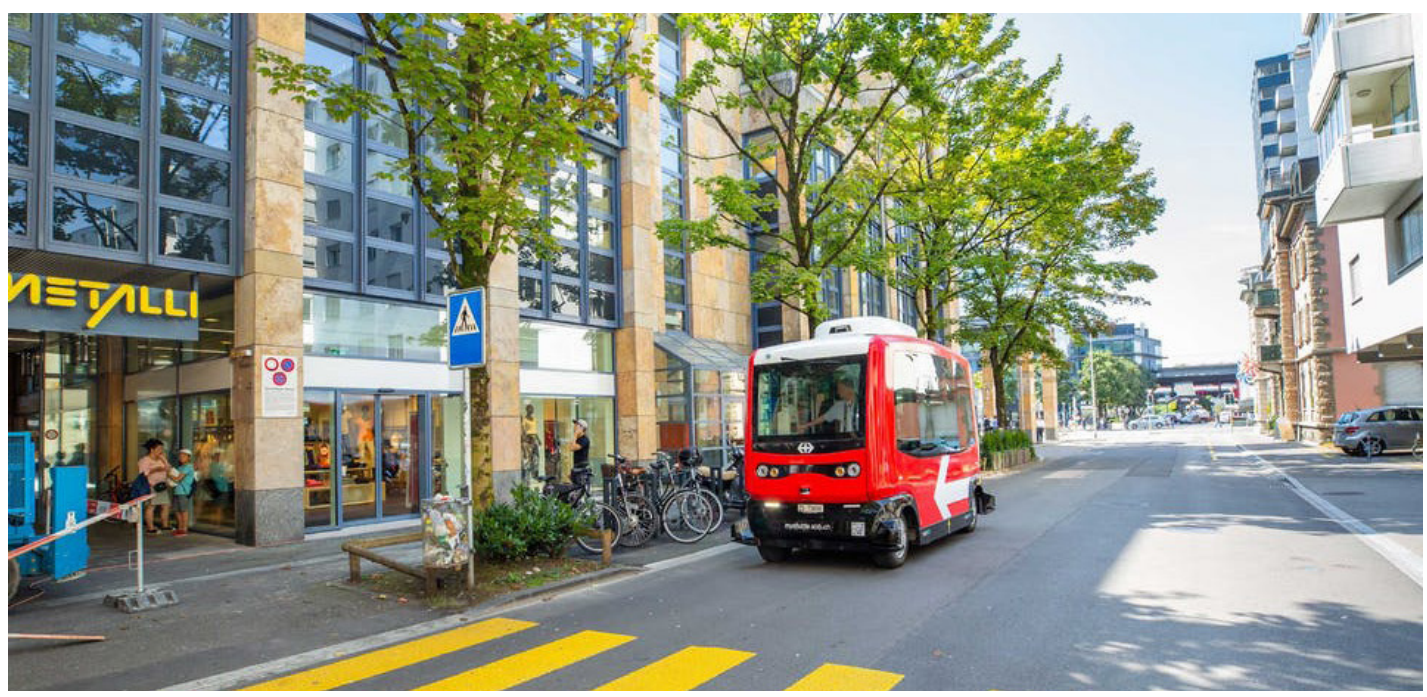

Quelle: SBB (2020: 1)

Im Rahmen des Projekts wurde der automatisierte Shuttlebus hauptsächlich zum Bedienen der ersten und letzten Meile innerhalb der Stadt Zug, insbesondere zwischen dem Bahnhof und den Arealen des Unternehmens V-Zug, eingesetzt. Abbildung 9 gibt einen Überblick der Routenführungen für den Einsatz des Shuttlebusses in der Stadt Zug. Während die Routenführungen 1 bis 4 allein für Funktionstests des Fahrzeugs oder kurze Demonstrationszwecke genutzt wurden, fand der regelmäßige und öffentliche Betrieb des automatisierten Shuttlebusses auf der Routenführung 5 (rosa) zwischen dem Bahnhof bzw. dem Einkaufszentrum Metalli sowie den Arealen des Unternehmens V-Zug oder auf den Routenführungen 6 und 7 (lila und grün), die jeweils eine adaptierte, zum Teil verkürzte Form der Routenführung 5 darstellen, statt (Abb. 9). 
EasyMile, der Hersteller des automatisierten Shuttlebusses, arbeitete mit vollem Einsatz daran, den Anforderungen des Projekts gerecht zu werden. Dank den großen Anstrengungen konnte man schließlich im Mischverkehr zwischen dem Bahnhof und den Arealen des Unternehmens V-Zug fahren. Allerdings gab es im Betrieb des automatisierten Shuttles immer wieder Herausforderungen: Das fehlerfreie Erkennen von Ampelzeichen, das Antizipieren des Verkehrsflusses, die Umfahrung von Hindernissen, das Fahren bei starkem Niederschlag oder selbstständiges "Lernen“ aus neuen Fahrsituationen waren durch das Fahrzeugmodell im Rahmen des Betriebs noch nicht möglich. Sensorstörungen durch Pollen, das Befahren von Kreuzungen mit Linksabbiegen, Störungen aufgrund wachsender Vegetation oder Baustellen waren Gegebenheiten, die eine Begleitung durch Sicherheitsfahrerlnnen unabdingbar machten. Aufgrund

Abbildung 9: Überblick der Routenführungen des automatisierten Shuttles in der Stadt Zug

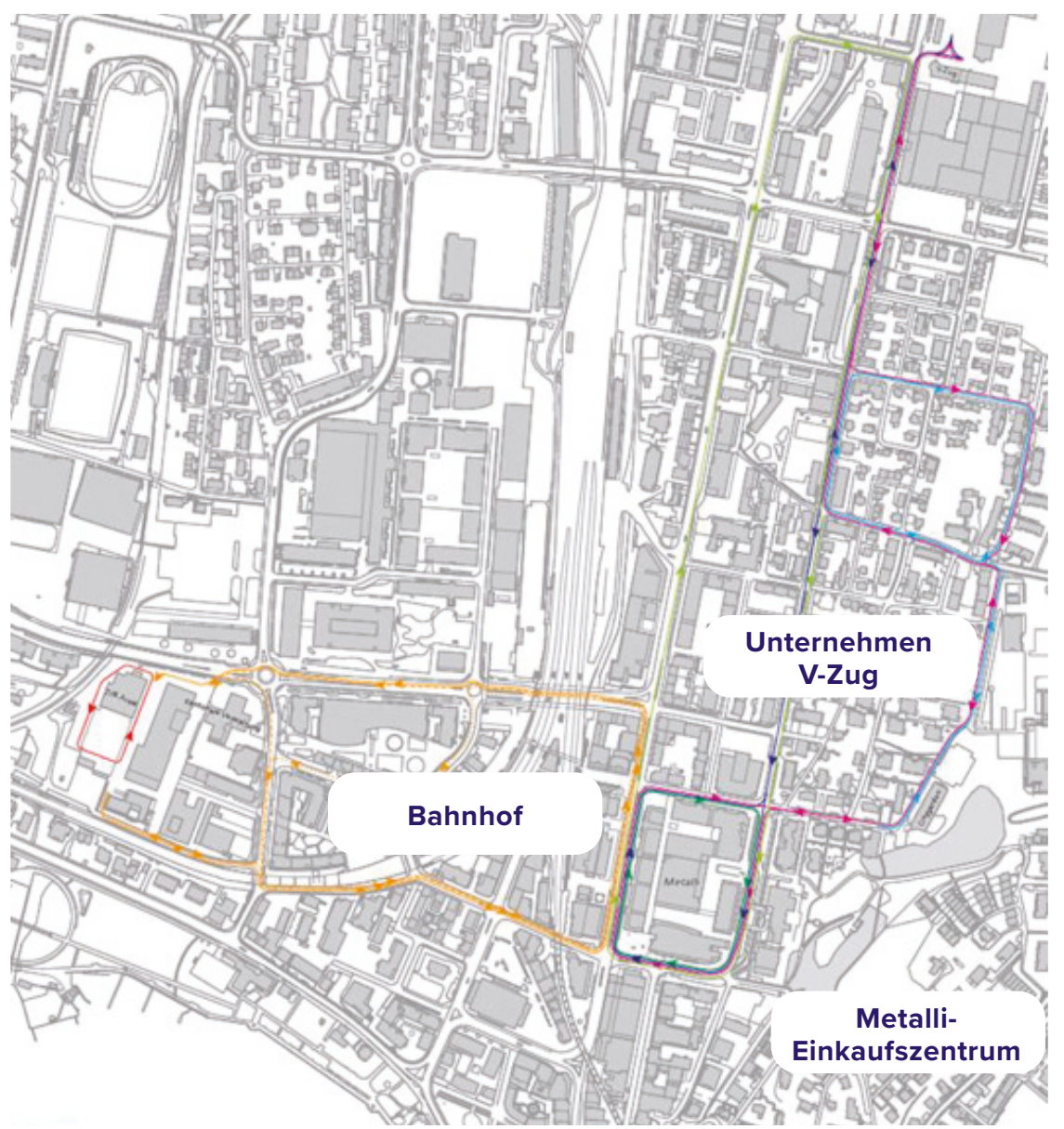

\begin{tabular}{l}
\hline Route 1 \\
\hline----- Route 2 - Option A \\
Route 2 - Option B \\
\hline Route 2 - Option D \\
Route 3 \\
Route 4 \\
Route 5 \\
Route 6 \\
Route 7 \\
Eigentum Stadt Zug \\
Eigentum Kanton Zug
\end{tabular}

Testroute ZVB-Areal

Hin- und Rückfahrt ZVB-Areal

Hin- und Rückfahrt ZVB-Areal

Hin- und Rückfahrt ZVB-Areal (während Baustelle)

Short-Loop Metalli Einkaufszentrum

City Garden Testroute

Metalli - V-Zug - Metalli (30er Zone)

Metalli - V-Zug - Metalli (via Industriestraße)

Metalli - V-Zug - Metalli (via Baarerstraße)

Quelle: SBB (2020: 12) 
der technischen Komplexität war es für EasyMile im Rahmen des Betriebs des Fahrzeugs im Projekt zudem nicht möglich, den Kundlnnen einen bedarfsgesteuerten Gebietsservice anzubieten (On-Demand-Angebot in einem ausgewählten Gebiet, in dem Passagierlnnen an jedem beliebigen Ort zu- und aussteigen können). Der Zu- und Ausstieg von Personen war im Betrieb nur bei Zwischenstopps an fixen Haltestellen entlang der Routenführung möglich.

\subsection{INTEGRATION IN KUNDENINFORMATIONSSYSTEME DES ÖFFENTLICHEN VER- KEHRS}

Ein wesentlicher Fokus des Projekts lag darin, eine Integration des Shuttlebusses in die Kundeninformationssysteme der SBB und demzufolge in die der bestehenden ÖV-Landschaft der Schweiz auszuloten. Hierzu wurde mit dem Unternehmen Bestmile, welches Dispositions- bzw. Flottenmanagementsysteme für automatisierte Fahrzeuge anbietet, zusammengearbeitet. Ziel war es, den automatisierten Shuttlebus in die verschiedenen Kundeninformationssysteme des öffentlichen Verkehrs, beispielsweise (1) in die Anzeige von Abfahrtszeiten an Monitoren an Bushaltestellen, (2) in die Anzeige von Anschlussverbindungen im Zug oder (3) in die Anzeige von Abfahrtszeiten in den SBB Apps, zu integrieren.

Die Kundeninformationssysteme dienen dazu, die Passagierlnnen über das Angebot bzw. deren Änderungen zu informieren. Die Schweiz ist in diesem Thema für den ÖV Vorreiter, weil die SBB im Auftrag des Bundesamts für Verkehr Informationen zur Mehrzahl der ÖV-Angebote bündelt und der Branche sowie den KundInnen zur Verfügung stellt.

Stark vereinfacht dargestellt sind diese Systeme auf aktuelle ÖV-Angebotsformen, d. h. auf im Vorfeld festgelegte Linienführungen mit fixen Haltestellen und entsprechenden langfristigen Fahrbzw. Zeitplänen ausgerichtet. Folgen Services mit automatisierten Fahrzeugen dieser Angebotslogik, wie der im Projekt implementierte liniengebundene Shuttlebus mit fixen Haltestellen, können sie in der aktuellen Systemlandschaft problemlos integriert und den Kundlnnen dargestellt werden. Ausschlaggebend ist dabei nicht der Automatisierungsgrad der Fahrzeuge, sondern ob das automatisierte Fahrzeug nach Fahrplan oder auf Abruf angeboten wird. Innerhalb des Projekts wurden so die Anschlussverbindungen des öffentlichen Verkehrs im Shuttlebus auf einem Monitor dargestellt (Abb. 10, links). Andererseits wurden ebenso die geplanten Abfahrtszeiten des Shuttlebusses vom Hauptbahnhof Zug an den Abfahrtsanzeigen am Bahnhof gezeigt (Abb. 10, rechts). Hierzu wurde der als klassischer Linienverkehr betriebene Shuttlebus als Linie 17 in das ÖV-Fahrplan- und Kundeninformationssystem integriert und ein Fahrplan des Shuttlebusses hinterlegt.

Abbildung 10: Anzeige der Anschlussverbindungen im Shuttlebus (links) und Anzeige des Shuttlebusses beim Abfahrtsmonitor im Bahnhof Zug (rechts)
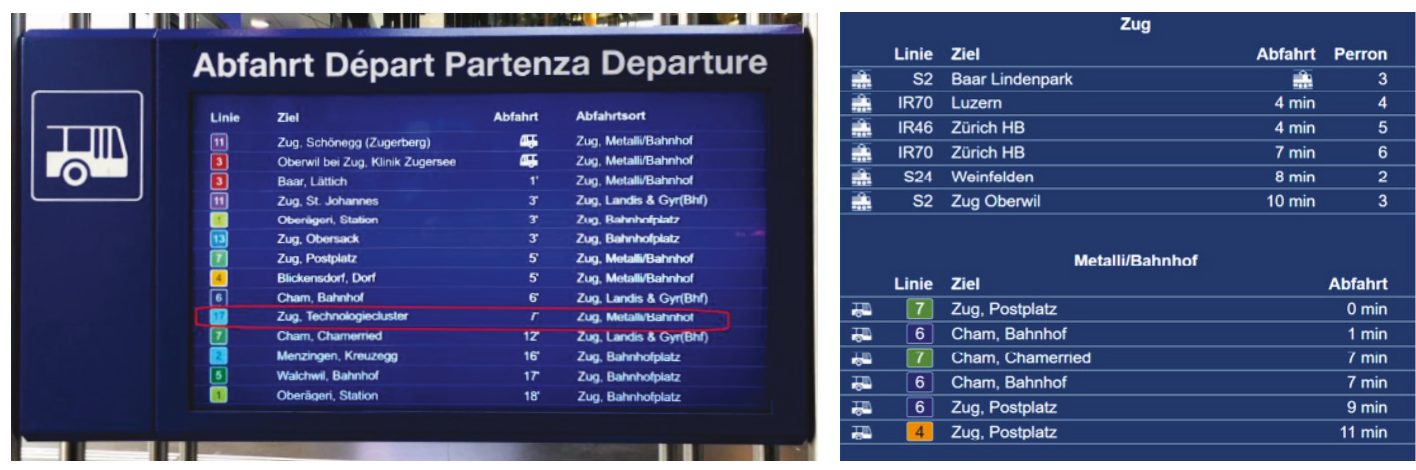

Quelle: SBB (2020: 32) 
Wie eingangs erwähnt, bieten automatisierte Fahrzeuge eine große Chance für neue Angebotsformen, insbesondere Transportservices auf Abruf (On-Demand-Services). Aufgrund seiner Automatisierung kann ein automatisiertes Fahrzeug zu einer bestimmten Zeit an einen definierten Ort bestellt werden, wobei es möglich ist, ohne Umstieg an den Zielort gebracht zu werden. Durch ein solches Angebot kann man auf den Besitz eines eigenen Autos verzichten. Wenn gleichzeitig verschiedene Fahrten aus Effizienzgründen gebündelt werden, vereint diese neue Angebotsform die Vorteile des öffentlichen Verkehrs mit denen des eigenen Fahrzeuges. Daraus entsteht der sogenannte öffentliche Individualverkehr (ÖIV).

Um den starken Schweizer ÖV mit diesen neuen Angebotsformen anzureichern, wurden im Pilotprojekt „MyShuttle“ erste Versuche unternommen, ein derartiges ÖIV-Angebot, wie beispielsweise ein Shuttlebus mit flexiblen Haltewünschen, in die Kundeninformation zu integrieren. Die hierfür notwendige Informationskette zwischen dem vorhandenen Kundeninformationssystem der SBB, dem Dispositions- bzw. Flottenmanagementsystem von Bestmile und dem Fahrzeug ist in Abbildung 11 ersichtlich. Der in den Systemen des ÖV enthaltene Fahrplan wird an das Steuerungs- bzw. Dispatchingsystem übermittelt, das den Fahrplan in Einzelfahrten aufteilt und sequenziell an das Fahrzeug weitergibt (Mission Management). Das Fahrzeug meldet stets Position, Geschwindigkeit und weitere Daten an das Dispatchingsystem von Bestmile zurück. Das Steuerungs- und Dispatchingsystem berechnet daraufhin das Delta zwischen Echtzeit- und Sollfahrplan und meldet es dann dem Kundeninformationssystem zurück, um letztendlich die Kundlnnen über die Abweichung zu informieren.

Abbildung 11: Schematische Darstellung der Informationskette zwischen Kundeninformationssystem, Dispatchingsystem und Fahrzeug
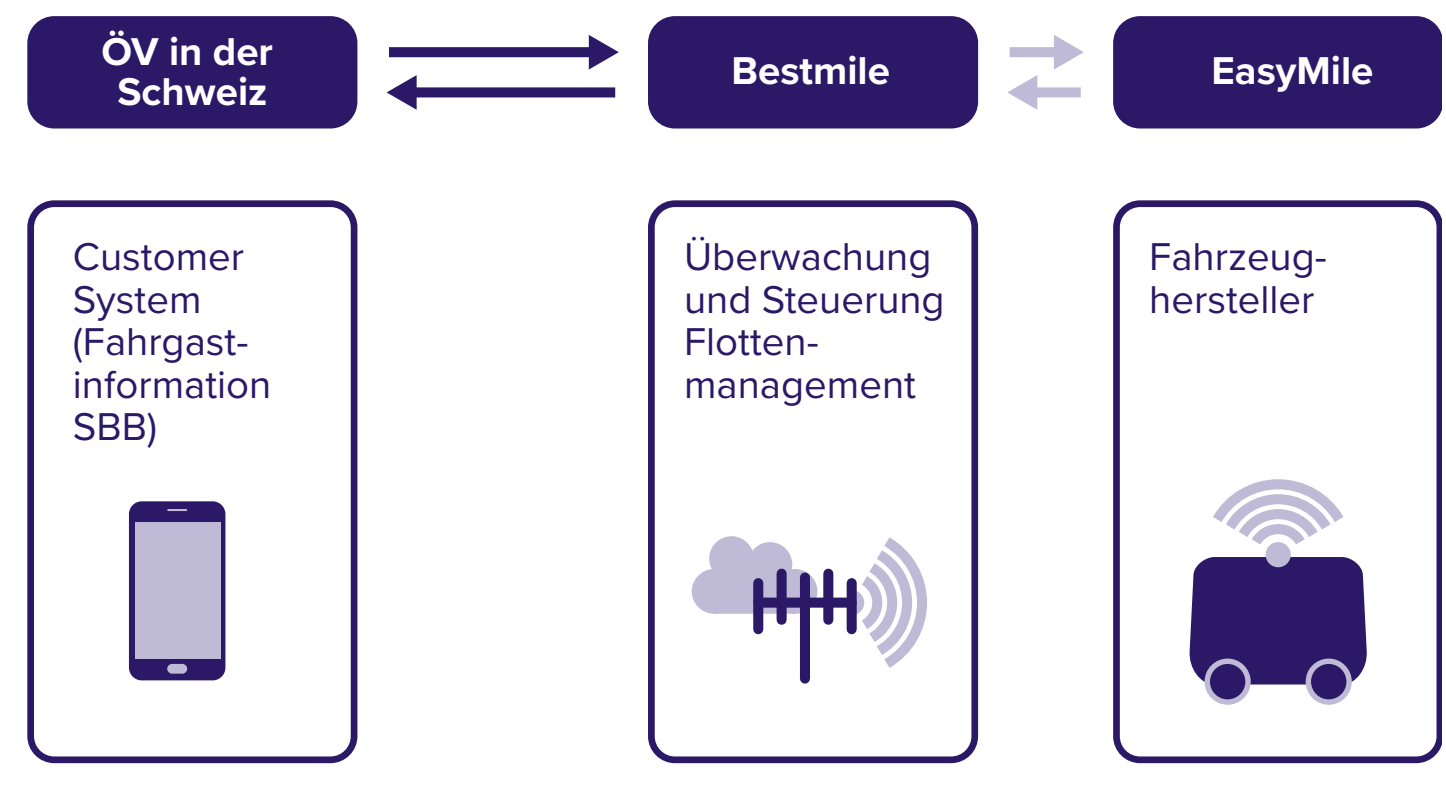

Quelle: eigene Darstellung nach SBB (2020: 32)

Ein wesentlicher Aspekt ist also, dass das Dispatchingsystem Fahraufträge, sogenannte Missions, an das Fahrzeug sendet, die es dann durchführt. Hierbei muss das Dispatchingsystem auch in der Lage sein, Veränderungen der Mission durchzuführen, beispielsweise durch einen verlangten Halt des Shuttlebusses, der während der Fahrt betätigt wird. Aufgrund der technischen Komplexität war es für EasyMile im Rahmen des Pilotbetriebs des Shuttlebusses nicht möglich, eine Schnittstelle zur Verfügung zu stellen, die eine Mission verarbeiten und mit Anpassungen der Mission umgehen kann, sowie einen bedarfsgesteuerten Gebietsservice, bei 
dem der/die Kundln an jedem beliebigen Ort zu- und aussteigen kann, anzubieten. Laut EasyMile sei der externe Zugriff auf das Fahrzeug durch eine Mission nach jetzigem Stand aus Sicherheitsgründen kritisch und könnte missbraucht werden.

Um die Integration solcher Angebotsformen in das bestehende Kundeninformationssystem dennoch ausloten zu können, wurden in der Folge (1) für einen „Proof of Concept“ Fahrzeuge im Dispatchingsystem von Bestmile fiktiv „erstellt“, also simuliert, und (2) mittels einer von Bestmile entwickelten App (Stadt Zug On-Demand-App) für Endkundlnnen die Funktionalität von flexiblen Halten des Shuttlebusses über die SicherheitsfahrerInnen untersucht.

Für (1) den „Proof of Concept“ wurde daran gearbeitet, eine Verbindung zwischen dem Kundeninformationssystem der SBB und dem Dispatchingsystem von Bestmile herzustellen. Dies war erforderlich, da die Formate des Kundeninformationssystems derzeit auf fixen Haltestellen und vordefinierten Linienführungen und Fahrplänen basieren, während das Dispatchingsystem für einen Gebietsservice, der nach Bedarf und nicht nach Fahrplan fährt, ausgelegt ist. Diese Tests wurden an Testsystemen durchgeführt, die tatsächlichen Produktivsysteme bzw. Kundeninformationssysteme der SBB standen dafür nicht zur Verfügung.

Für die Tests wurden das Bestmile-System und das Kundeninformationssystem der SBB (CUS - Customer System) mit einem Adapter, der die Logiken beider Systeme zusammenbringt, verbunden und in weiterer Folge mit einem Router (MetaRouter) sowie den Informationskanälen der SBB MIKU (Mobiles Informationstool für Kundenkontakte) oder der KIB (Kundeninformation am Bahnhof, d. h. Lautsprecher und Bildschirme) verbunden (Abb. 12).

Das CUS erreichte hier jedoch seine Grenzen. Solange man sich im Rahmen des Linienverkehrs bewegte, konnten die durch Bestmile simulierten Fahrzeuge erfolgreich in den Kerninformationssystemen des öffentlichen Verkehres angezeigt werden (siehe hierzu auch die bereits

Abbildung 12: Informationsfluss für die ÖV-Integration und den „Proof of Concept“ und damit den Adapter zwischen Customer System (CUS) der SBB und Bestmile

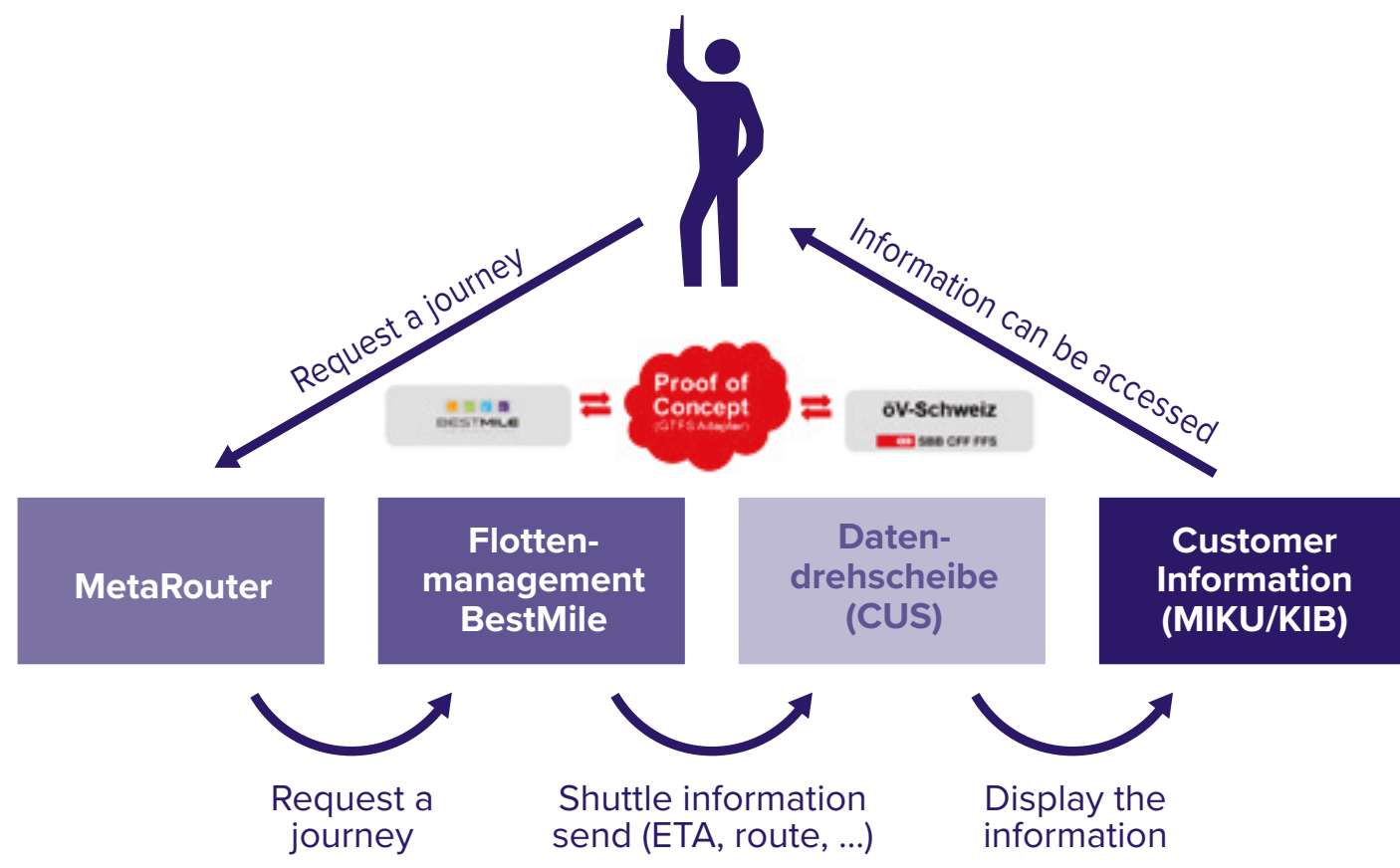

Quelle: eigene Darstellung nach SBB (2020: 26) 
erwähnte erfolgreiche Implementierung des liniengebundenen Shuttlebusses in die Abfahrtsanzeigen am Bahnhof). Eine vorgeschaltete Logik erkannte und stellte sogar gebündelte Fahrten (Bündelung von mehreren Buchungen aus dem MetaRouter auf ein Fahrzeug) sinnvoll dar. Sobald man jedoch den Linienverkehr verließ und auf Gebietsservice ohne Fahrpläne ausweitete bzw. versuchte, dieses zu simulieren (Fahrt und Halt auf Verlangen), konnte das System die Information nicht mehr verarbeiten und anzeigen.

Für (2) die Erprobung der Funktionalität flexibler Haltewünsche wurde eine App für die Stadt Zug von Bestmile entwickelt (Zug On-Demand), wobei Haltewünsche über die App allein an den/die Sicherheitsfahrerln übermittelt wurden. Diese Haltewünsche erschienen dann in der Driver-App des/der Sicherheitsfahrerln. Diese/r konnte die gemeldeten Wünsche manuell annehmen oder ablehnen (Abb. 13).

Abbildung 13: "Zug On-Demand"-App (oben) und Driver-App-Interface der Fahrt- und Haltewünsche (unten)

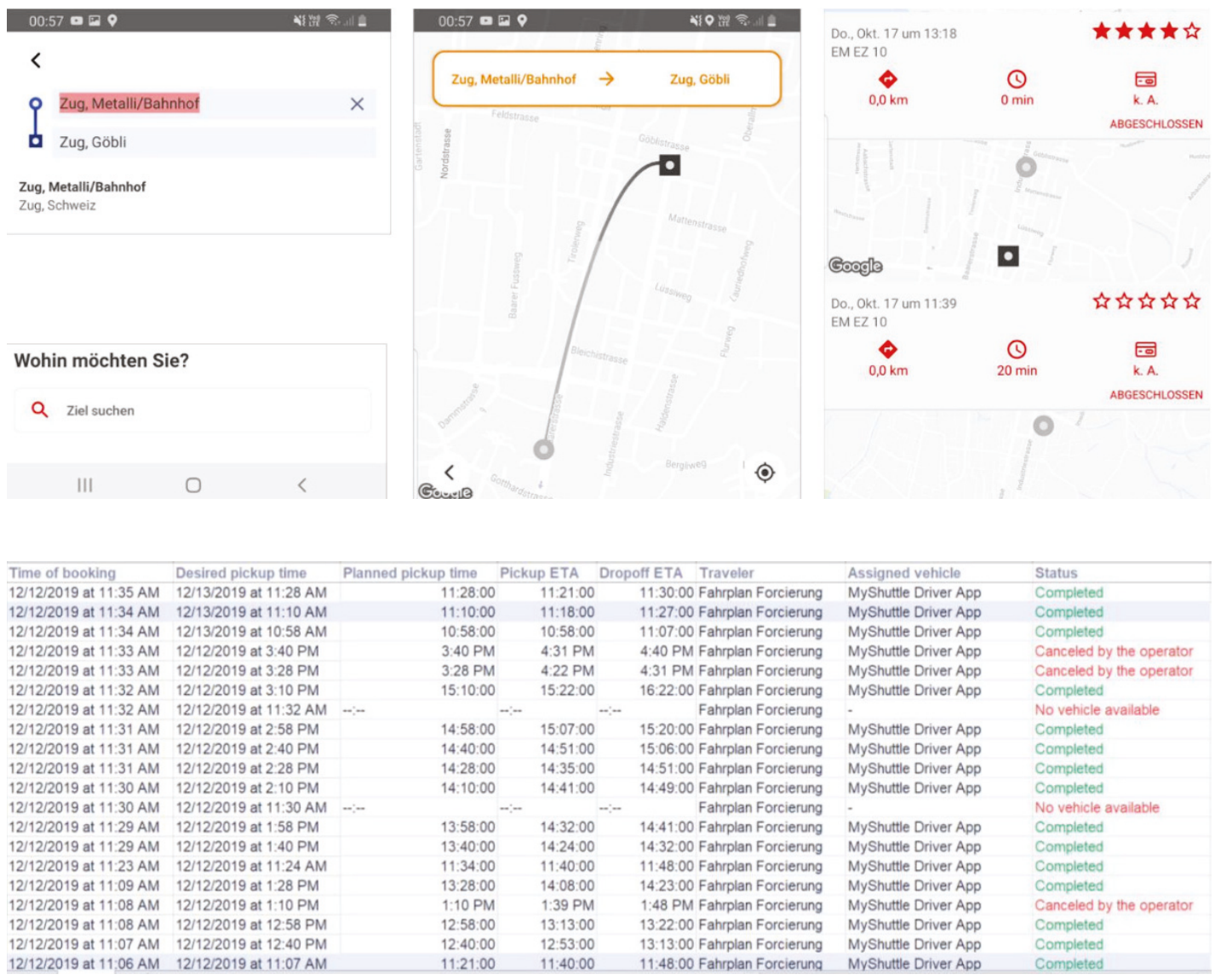

Quelle: SBB (2020: 32)

Die Verwendung der App zur Übermittlung von Haltewünschen an den/die Sicherheitsfahrerln half zwar, die Funktionalität flexibler Haltewünsche zu erproben, es zeigten sich aber - insbesondere aufgrund der schlanken Ausgestaltung der App, die der kurzen Entwicklungszeit geschuldet war - einige Probleme: Beispielsweise wurden Adressen nicht korrekt erkannt und zeitweise falschen Haltestellen zugeordnet, sodass Kundlnnen lange und nicht notwendige Fußwege zurücklegen mussten. Hinzu kam, dass die Destination nicht während der Fahrt verändert werden konnte, sondern nur bei Stillstand. Somit war es möglich, dass Kundlnnen einer 
Fahrt zugeteilt waren, aber das Fahrzeug, das schon unterwegs war, nicht anhielt. Insgesamt konnten so rudimentäre On-Demand-Funktionalitäten im Rahmen des Projekts abgebildet werden, die von Bestmile zur Verfügung gestellte Software besaß zum Zeitpunkt des Projekts aber nicht den benötigten Reifegrad.

\subsection{BISHERIGE ERKENNTNISSE}

Insgesamt hat das Projekt zwar nicht alle ursprünglich formulierten Ziele erreicht, die übergeordneten Lernziele wurden dennoch übertroffen. Innerhalb der SBB konnte wesentliches Know-how im Bereich automatisierten Fahrens, dessen Kundenakzeptanz und insbesondere in der Integration in das ÖV-System aufgebaut werden. Um automatisierte Shuttlebusse als neue Angebotsform im Kontext des öffentlichen Verkehrs zu etablieren, sollten die Buchbarkeit und die Kundeninformation der Shuttlebusse über klassische ÖV-Kanäle (z. B. SBB-App) möglich sein. Auch muss der Einsatz von bedarfsgerechten, flexiblen Shuttlebussen an die Ankunfts- oder Abfahrtszeiten des übergeordneten ÖV geplant sein. Im Rahmen des Tests zeigte sich jedoch, dass aktuelle Kernsysteme des ÖV, die auf feste Haltestellen und Linien sowie einen fixen Fahrplan ausgerichtet sind, noch nicht mit Bedarfsverkehr (ohne fixen Fahrplan), Kursänderungen oder flexiblen Halten, sondern allein mit Shuttlebussen, die im Linienverkehr verkehren, umgehen können. Hierbei bedarf es neuer Systeme für die Integration und die Entwicklung standardmäßiger APIs („application programming interface“/Programmierschnittstellen), die von den ÖV-Kernsystemen verarbeitet werden können.

Insgesamt konnte mit dem Projekt jedoch rechtzeitig wichtiges Wissen über neuartige Angebotsformen aufgebaut werden, um zum Zeitpunkt der Marktreife der Automatisierungstechnologie gegebenenfalls einen volkswirtschaftlich optimierten, kundenorientierten Service für die Schweiz anbieten zu können. Insbesondere die Ergebnisse des Projekts hinsichtlich der Integration automatisierter Shuttlebusse bzw. automatisierter On-Demand-Services in die Kundeninformationssysteme der SBB bzw. des öffentlichen Verkehrs werden nun dazu genutzt, um die genannten Systeme für diese neue Art von Mobilität fit zu machen.

\section{FAZIT}

Automatisiertes Fahren ermöglicht vielfältige Einsatzmöglichkeiten für neue Angebotsformen im öffentlichen Verkehr, die in das bestehende öffentliche Verkehrssystem integriert werden könnten. Durch eine Konvergenz des traditionellen öffentlichen Verkehrs und individuellerer Angebotsformen mit dem Fokus auf Sharing und Mitfahrservices kann der öffentliche Verkehr zukünftig personalisierter und flexibler gestaltet sowie eine zeitliche und räumliche Verdichtung des Angebots erreicht werden. Damit könnte letztlich den Bedürfnissen von Kundlnnen breiter und besser entsprochen werden.

Um die Anforderungen für einen bestmöglichen Einsatz neuer, automatisierter Angebotsformen zu erproben, braucht es umfangreiche Testbetriebe. Nicht nur hinsichtlich der technologischen Funktionsweise und Weiterentwicklung automatisierter Fahrzeuge, sondern auch um Erfahrungen zur Integration der Fahrzeuge in das bestehende öffentliche Verkehrssystem und zu den Wechselwirkungen mit den lokalen Gegebenheiten zu sammeln. Im Fokus der derzeitigen Auseinandersetzung des öffentlichen Verkehrs mit automatisierten Fahrzeugen stehen Testprojekte, in denen der Betrieb und die Integration von automatisierten Shuttlebussen er- 
probt wird. Diese Testprojekte sind zum Teil in unterschiedlichen Gebietstypen bzw. räumlichen Settings (z. B. Stadtrand einer Großstadt, Kleinstadt, Dorf) verortet, weisen unterschiedliche Betriebskonzepte auf und stellen auch unterschiedliche Aspekte (z. B. technologische, organisatorische und betriebliche sowie wirtschaftliche Machbarkeit) stärker oder weniger stark in den Vordergrund. Oftmals geht mit den Testprojekten für die beteiligten Akteure wie öffentliche Verkehrsbetriebe bzw. -unternehmen oder Städte und Kommunen auch eine gewisse Demonstration von Innovationsfähigkeit und ein zukunftsfähiges Image einher (vgl. Perkins et al. 2018: 10). Allerdings konnten die beispielhaften Vertiefungen deutlich machen, dass es für die beteiligten Akteure auch noch weitere Vorteile und Potentiale gibt, sich an der Erforschung zukünftiger Mobilitätsoptionen im öffentlichen Verkehr zu beteiligen und in der Praxis zu erproben. So können Testprojekte mit automatisierten Fahrzeugen bzw. speziell auch mit automatisierten Shuttlebussen im öffentlichen Verkehr dienlich sein, um

- mögliche Betriebskonzepte in der Praxis zu prüfen,

- unterschiedlichen StakeholderInnen zu ermöglichen, hierzu Erfahrungen zu sammeln,

- Nutzerlnnen in den Betrieb zu involvieren und deren Akzeptanz zu eruieren,

- Know-how hinsichtlich des Betriebs, der Reparatur und des Service aufzubauen,

- $\quad$ eine entsprechende Dateninfrastruktur (z. B. operative und analytische Datensätze, Definition von Anforderungen für den Datentransfer, Schnittstellen etc.) zu erstellen, die für die Evaluierung bisheriger Testbetriebe und zukünftige Vorhaben relevant sein wird.

Die beiden Testprojekte „autoNV OPR“ und „MyShuttle“ zeigen auf, dass die genutzten automatisierten Shuttlebusse im Betrieb noch an ihre Grenzen stoßen; dies betrifft in erster Linie Aspekte der technologischen sowie der organisatorischen und betrieblichen Machbarkeit:

\section{Technologische Machbarkeit}

- Ein störungsfreier Einsatz bzw. Betrieb der Shuttlebusse ist ohne Anpassungen der Infrastruktur (z. B. Aufstellung von künstlichen Landmarkern in Form von Schildern, Einführung eines Halteverbots etc.) derzeit herausfordernd.

- Speziell an komplexen Knotenpunkten (insbesondere mit Linksabbiegen) muss die Fahrt häufig durch die OperatorInnen freigegeben werden, damit der Shuttlebus diese passieren kann. Probleme bestehen auch im fehlerfreien Erkennen von Ampelzeichen, hinsichtlich des Antizipierens des Verkehrsflusses, bezüglich der Umfahrung von Hindernissen, aufgrund von (Sensor-)Störungen durch Pollen, wachsender Vegetation und Baustellen oder beim Fahren bei starkem Niederschlag.

- Überholvorgänge anderer Verkehrsteilnehmerlnnen, gefolgt von engem Einscheren vor dem Shuttlebus, führen zu von den Fahrgästen als negativ empfundenen Verzögerungen.

\section{Organisatorische und betriebliche Machbarkeit}

- Die Integration der automatisierten Shuttlebusse in aktuelle Kernsysteme des öffentlichen Verkehrs ist derzeit nur gut möglich, wenn diese im Linienverkehr (fixer Fahrplan und Halte) verkehren. Bedarfsgerecht verkehrende automatisierte Shuttlebusse mit flexiblen Halten sind in derzeit bestehenden ÖV-Kernsystemen kaum umsetzbar. 
Insgesamt lässt sich festhalten, dass es sich durchaus für den öffentlichen Verkehr sowie städtische Akteure lohnt, finanzielle Ressourcen in das Testen automatisierter Fahrzeuge bzw. in Testprojekte mit automatisierten Shuttlebussen zu investieren, da damit neue Erkenntnisse und wichtiges Know-how aufgebaut werden kann. Um die automatisierten Shuttlebusse jedoch tatsächlich als vollwertige Mobilitätsoption zur Ergänzung des bestehenden öffentlichen Verkehrsangebots z. B. in Gebieten geringer Nachfrage einsetzen zu können, bedarf es, unter Berücksichtigung der Ergebnisse aus den Beispielprojekten, folgender Rahmenbedingungen bzw. Maßnahmen:

1. Weiterentwicklung der Fahrzeugtechnologie, um einen stabilen Betrieb und eine höhere Fahrgeschwindigkeit zu ermöglichen: Eine intelligentere, antizipativere Fahrzeugtechnologie ist für den Betrieb des automatisierten Shuttlebusses notwendig, um etwaige Verzögerungen und Eingriffe der Operatorlnnen zu reduzieren und einen stabilen Betrieb zu realisieren. Die Fahrgeschwindigkeit des automatisierten Shuttlebusses bildet dabei einen wichtigen Aspekt für die Nutzung, die Attraktivität und den Betrieb des Fahrzeugs und sollte mit einer Weiterentwicklung der Fahrzeugtechnologie erhöht werden. Dieser Sachverhalt ist weniger in der Feinerschließung von kleineren Wohngebieten wichtig, in welchen die automatisierten Shuttlebusse nicht unbedingt mit einer hohen Geschwindigkeit verkehren müssen, sondern vor allem in Fällen, in denen hierdurch größere Gebiete erschlossen werden sollen bzw. Einrichtungen oder Orte in größerer Entfernung verbunden werden müssen und die automatisierten Shuttlebusse tendenziell auf höherrangigen Straßen unterwegs sind.

2. Keine umfangreiche Anpassung der Infrastruktur bzw. eine umfangreiche Anpassung der Infrastruktur nur dann, wenn auch andere Verkehrsmodi (z. B. Fahrrad, Zufußgehen) profitieren: Eine Anpassung der Infrastruktur, die bereits heute im Zuge von Tests mit automatisierten Shuttlebussen in geringem Ausmaß durchgeführt wird, sollte auch weiterhin nur in geringem Ausmaß zur Ermöglichung der Tests erfolgen. Auf umfassende infrastrukturelle Anpassungen (sowohl baulich als auch digital, z. B. „vehicleto-infrastructure“ etc.) sollte verzichtet werden, da sich einerseits die infrastrukturellen Anforderungen der Fahrzeuge rasch ändern können und gleichfalls die Kosten für eine Infrastrukturanpassung relativ hoch ausfallen. Hinzu kommt, dass bereits heute der öffentliche Raum durch Nutzungskonflikte geprägt ist und auf eine weitere Flächeninanspruchnahme bzw. ein Verstellen des öffentlichen Raums durch Infrastruktur für automatisierte Shuttlebusse verzichtet werden sollte. Eine umfangreiche Anpassung der Infrastruktur sollte somit allenfalls dann erfolgen, wenn hiervon nicht nur die automatisierten Shuttlebusse, sondern auch der Fuß- und Radverkehr profitieren.

3. Vertiefende Berücksichtigung der Verknüpfung von automatisierten Angebotsformen und des Umweltverbunds (öffentlicher Verkehr, Fahrrad, Zufußgehen): Die Verknüpfung von automatisierten (und vernetzten) Angebotsformen mit anderen Verkehrsmodi, insbesondere dem bestehenden öffentlichen Verkehr, ist essentiell. Für die Integration bedarfsgerecht verkehrender automatisierter Shuttlebusse mit flexiblen Halten in bestehenden ÖV-Kundeninformations- oder Buchungssystemen sind neue Systeme und standardmäßige Schnittstellen bzw. APIs, die von den ÖV-Kernsystemen verarbeitet werden können und an die sich Anbieter halten, erforderlich. Vor dem Hintergrund des Komforts für die Kundlnnen sollte die Buchung von Fahrten möglichst einfach und vorwiegend digital erfolgen. Besonderes Augenmerk muss auch auf eine kompatible Anschlusssicherung zwischen dem übergeordneten ÖV und dem automatisierten Shuttlebus gelegt werden (Orientierung des Einsatzes von bedarfsgerechten, flexiblen Shuttlebussen an den Ankunfts- oder Abfahrtszeiten des übergeordneten ÖV; vgl. SBB 2020: 62ff.). 
Darüber hinaus sind noch weitere Rahmenbedingungen bzw. Maßnahmen von wichtiger Bedeutung:

4. Orientierung der Betriebskonzepte an der Verkehrsnachfrage und Siedlungsstruktur des jeweiligen Gebietes: Der Einsatz automatisierter Shuttlebusse sollte in seiner Ausgestaltung, d. h. hinsichtlich Betriebsdauer, Takt, Route, Bediengebiet etc., immer an die Verkehrsnachfrage und die Siedlungsstruktur des jeweiligen Gebietes orientiert werden. Hierbei kann es sinnvoll sein, den Einsatz an die in den jeweiligen Verkehrsentwicklungsplänen beschriebenen Ansprüche und Ziele zu knüpfen und darin zu integrieren.

5. Räumliche Integration mit bestehendem ÖV-Angebot: Eine Integration bzw. Verknüpfung mit dem bestehenden ÖV-Angebot ist nicht nur aus technologischer Sicht erforderlich, sondern auch räumlich: Hierbei sollte der Fokus auf flexiblen und alternativen Angebotsformen als Zubringer des klassischen Linienverkehrs und auf sogenannten „transit-oriented developments“ (TODs) liegen, d. h., insbesondere die funktionale Anreicherung und Siedlungsentwicklung im Stationsumfeld gefördert werden. Der Einsatz von automatisierten Shuttlebussen als Zubringer zur Schiene ist notwendig, um zu verhindern, dass automatisierte Privatfahrzeuge diese Funktion übernehmen (vgl. Sinner 2019): zum einen, da die Flächenerfordernisse an Bahnhöfen und Umstiegspunkten im Fall von Bedarfsbussen und Zubringerdiensten wesentlich geringer ausfallen als für automatisierte Privatfahrzeuge oder Car-Sharing-Dienste (vgl. Sinner et al. 2018), zum anderen, da durch einen räumlich erleichterten Umstieg zwischen Modi die gesamte Reisezeit verkürzt werden kann und intermodale Wege für Nutzerlnnen an Attraktivität gewinnen können (siehe Beitrag 8 von Bruck et al. in diesem Band).

6. Einbindung von relevanten StakeholderInnen und Partizipation: Im Rahmen weiterer Testprojekte mit automatisierten Shuttlebussen sollten die relevanten Stakeholderlnnen und AkteurInnen der Gemeinden und zugehörigen Verwaltungseinheiten eingebunden werden. Darüber hinaus sollten ebenso Partizipationsmöglichkeiten für die örtliche Bevölkerung geschaffen werden, um den Forschungs- und Entwicklungsprozess entsprechend den lokalen Anforderungen und Zielen auszurichten.

Zusätzlich besteht weiterer Forschungsbedarf rund um den Einsatz von automatisierten Fahrzeugen bzw. insbesondere automatisierten Shuttlebussen im öffentlichen Verkehr hinsichtlich folgender Aspekte, die im Rahmen weiterer Testprojekte untersucht werden sollten:

- Kosten: Untersuchungen zur möglichen Kostenreduktion durch den Einsatz automatisierter Fahrzeuge und zu möglicherweise neu anfallenden Kosten (z. B. Dispositionssysteme, Begleitpersonal, Schutz vor Vandalismus, häufigere Reinigungsarbeiten etc.).

- Infrastruktur: Dabei handelt es sich um erforderliche Infrastrukturen nicht nur für den Betrieb der Fahrzeuge im Speziellen, sondern insbesondere auch für deren größeren betrieblichen Einsatz, z. B. hinsichtlich Ladeinfrastruktur, an Haltestellen (Verkehrsflächen) etc.

- Nutzerakzeptanz: Die Akzeptanz durch die NutzerInnen ist nicht nur hinsichtlich der Technologie an sich relevant (z. B. Mitfahren im Shuttlebus, Komfort, Qualität), sondern im Spezifischen auch in der Nacht (Sicherheitsgefühl) bzw. hinsichtlich der Frage, ob Begleitpersonal in gewissen Zeiten benötigt wird.

- Dimensionierung der Flotten: Es sind Untersuchungen zur Ausgestaltung der notwendigen automatisierten Fahrzeugflotte, z. B. auch Bündelungspotential, Routen, Disposition etc., notwendig. 


\section{LITERATUR}

Ainsalu, J., V. Arffman, M. Bellone, M. Ellner, T. Haapamäki, N. Haavisto, E. Josefson, A. Ismailogullari, B. Lee, O. Madland, R. Madžulis, J. Müür, S. Mäkinen, V. Nousiainen, E. Pilli-Sihvola, E. Rutanen, S. Sahala, B. Schønfeldt, P. M. Smolnicki, R.-M. Soe, J. Sääski, M. Szymańska, I. Vaskinn und M. Åman 2018. „State of the Art of Automated Buses“, in Sustainability (10) 3118, 1-34.

Alessandrini,A.2016. „FinalReportSummary-CITYMOBIL2(Cities demonstrating cyberneticmobility)“. Download unter https://cordis.europa.eu/project/id/314190/reporting (24.7.2020).

Alessandrini, A., A. Campagna, P. Delle Site, F. Filippi und L. Persia 2015. „Automated Vehicles and the Rethinking of Mobility and Cities“, in Transport Research Procedia 5, 145-160.

Barillère-Scholz, M., C. Büttner und A. Becker 2020. „Mobilität 4.0: Deutschlands erste autonome Buslinie in Bad Birnbach als Pionierleistung für neue Verkehrskonzepte“, in Autonome Shuttlebusse im ÖPNV. Analysen und Bewertungen zum Fallbeispiel Bad Birnbach aus technischer, gesellschaftlicher und planerischer Sicht, hg. v. A. Riener, A. Appel, W. Dorner, T. Huber, J. C. Kolb und $\mathrm{H}$. Wagner. Wiesbaden: Springer Vieweg, 15-22.

BMVI (Bundesministerium für Verkehr und digitale Infrastruktur) 2016. „Mobilitäts- und Angebotsstrategien in ländlichen Räumen. Planungsleitfaden für Handlungsmöglichkeiten von ÖPNVAufgabenträgern und Verkehrsunternehmen unter besonderer Berücksichtigung wirtschaftlicher Aspekte flexibler Bedienungsformen“, Berlin. www.bmvi.de/SharedDocs/DE/Publikationen/G/ mobilitaets-und-angebotsstrategien-in-laendlichen-raeumen-neu.pdf?__blob=publicationFile (19.8.2020).

bmvit (Bundesministerium für Verkehr, Innovation und Technologie) 2019. „Nahverkehr. Recht“. Download unter: www.bmvit.gv.at/verkehr/nahverkehr/recht/index.html (20.1.2020).

Bösch, P. M., F. Becker, H. Becker und K. W. Axhausen 2018. „Cost-based analysis of autonomous mobility services“, in Transport Policy 64, 76-91. DOI: /10.1016/j.tranpol.2017.09.005.

Bruns, F., M. Rothenfluh, M. Neuenschwander, M. Sutter, B. Belart und M. Egger 2018. „Einsatz automatisierter Fahrzeuge im Alltag - Denkbare Anwendungen und Effekte in der Schweiz. Schlussbericht Modul 3c ,Mögliche Angebotsformen im kollektiven Verkehr (ÖV und ÖIV)““, definitive Fassung vom 19.4.2018. www.ebp.ch/sites/default/files/project/uploads/2018-04-19\%20 aFn_3c\%20Mögliche\%20Angebotsformen\%20im\%20kollektiven\%20Verkehr_Schlussbericht_0.pdf (18.8.2020).

Buffat, M., H. Sommer, M. Amacher, R. Mohagheghi, J. Beckmann und A. Brügger 2018. „Individualisierung des ÖV-Angebots. Analyse der Auswirkungen der Individualisierung und weiterer angebots- und nachfragerelevanten Trends auf die zukünftige Ausgestaltung des ÖV-Angebots“, Forschungsprojekt SVI 2014/004 auf Antrag der Schweizerischen Vereinigung der Verkehrsingenieure und Verkehrsexperten (SVI). Bern: Eidgenössisches Departement für Umwelt, Verkehr, Energie und Kommunikation UVEK.

Derer, M., und F. Geis 2020. „Entwicklungen im ÖPNV“, in: Autonome Shuttlebusse im ÖPNV. Analysen und Bewertungen zum Fallbeispiel Bad Birnbach aus technischer, gesellschaftlicher und planerischer Sicht, hg. v. A. Riener, A. Appel, W. Dorner, T. Huber, J. C. Kolb und H. Wagner. Wiesbaden: Springer Vieweg, 7-14.

EasyMile 2019a. „EZ 10“. https://easymile.com/solutions-easymile/ez10-autonomous-shuttle-easymile/ (2.11.2019).

EasyMile 2019b. „Autonomous Technology Thanks To A Unique \& Versatile Software Package“. https://easymile.com/driverless-technology-easymile-how-does-it-work/ (9.3.2020).

EPOMM - European Platform on Mobility Management 2017. „Die Rolle von Mobilität als Dienstleistung für Mobilitätsmanagement“, E-Update Dezember 2017. www.epomm.eu/newsletter/v2/ content/2017/1217_2/doc/eupdate_de.pdf (7.1.2020).

FGSV (Forschungsgesellschaft für Straßen- und Verkehrswesen) 2006. „Richtlinien für die Anlage von Stadtstraßen - RASt 06“, Ausgabe 2006. Download unter www.forschungsinformationssystem.de/servlet/is/232185/ (19.8.2020).

Földes, D., und C. Csiszár 2018. „Framework for planning the mobility service based on autonomous vehicles“, Konferenzpapier, Smart City Symposium Prague (SCSP) 2018. Prag. 
Gertz, C., und M. Dörnemann 2016. „Wirkungen des autonomen / fahrerlosen Fahrens in der Stadt Entwicklung von Szenarien und Ableitung der Wirkungsketten“. Bremen: Der Senator für Umwelt, Bau und Verkehr.

Hagenzieker, M., R. Boersma, P. Nuñez Velasco, M. Ozturker, I. Zubin und D. Heikop 2020. „Automated Buses in Europe. An Inventory of Pilots“, Version 0.5. TU Delft.

Heinrichs, D., S. Rupprecht und S. Smith 2019. „Making Automation Work for Cities: Impacts and Policy Responses“, in: Road Vehicle Automation 5, hg. v. G. Meyer und S. Beiker. Cham: Springer International Publishing, 243-252.

Hell, W. (Hrsg.) 2006. Öffentlicher Personennahverkehr. Herausforderungen und Chancen. Berlin/ Heidelberg: Springer-Verlag Berlin Heidelberg.

Hoadley, S. (Hrsg.) 2017. „Mobility as a service: Implications for urban and regional transport. Discussion paper offering the perspective of Polis member cities and regions on Mobility as a Service (MaaS)“, Polis Traffic Efficiency \& Mobility Working Group, September 2017. Brüssel. www.polisnetwork.eu/wp-content/uploads/2017/12/polis-maas-discussion-paper-2017-final_. pdf (19.8.2020).

Hörl, S. 2020. „Dynamic Demand Simulation for Automated Mobility on Demand“, Dissertation, ETH Zürich.

Hörl, S., F. Becker, T. Dubernet und K. W. Axhausen 2019. „Induzierter Verkehr durch autonome Fahrzeuge: Eine Abschätzung“, Forschungsprojekt SVI 2016/001 auf Antrag der Schweizerischen Vereinigung der Verkehrsingenieure und Verkehrsexperten (SVI). Bern: Eidgenössisches Departement für Umwelt, Verkehr, Energie und Kommunikation UVEK. https://ethz.ch/content/dam/ethz/ special-interest/baug/ivt/ivt-dam/vpl/reports/1401-1500/ab1433.pdf (18.8.2020).

Hörold, S. 2016. Instrumentarium zur Qualitätsevaluation von Mobilitätsinformation. Schriften zur Medienproduktion. Wiesbaden: Springer Vieweg.

Jittrapirom, P., V. Caiati, A.-M. Feneri, S. Ebrahimigharehbaghi, M. J. Alonso González und J. Narayan 2017. „Mobility as a Service: A Critical Review of Definitions, Assessments of Schemes, and Key Challenges“, in Urban Planning (2) 2, 13-25.

Jonuschat, H., A. Knie und L. Ruhrort 2016. „Zukunftsfenster in eine disruptive Mobilität. Teil 1: Mobilität in einer vernetzten Welt“. Berlin: Innovationszentrum für Mobilität und gesellschaftlichen Wandel (InnoZ). https://docplayer.org/57659946-Zukunftsfenster-in-eine-disruptive-mobilitaet. html (25.8.2020).

Jürgens, L. 2020. „Konnektivitätsveränderungen im ÖPNV-Netz durch die Einführung eines autonomen Shuttlebusses“, in: Autonome Shuttlebusse im ÖPNV. Analysen und Bewertungen zum Fallbeispiel Bad Birnbach aus technischer, gesellschaftlicher und planerischer Sicht, hg. v. A. Riener, A. Appel, W. Dorner, T. Huber, J. C. Kolb und H. Wagner. Wiesbaden: Springer Vieweg, 39-54.

Lenz, B., und E. Fraedrich 2015. „Neue Mobilitätskonzepte und autonomes Fahren: Potenziale der Veränderung", in Autonomes Fahren. Technische, rechtliche und gesellschaftliche Aspekte, hg. v. M. Maurer, J. C. Gerdes, B. Lenz und H. Winner. Berlin/Heidelberg: Springer Vieweg, 175-196.

Mitteregger, M., A. Soteropoulos und M. Berger 2019. „A Framework for Assessing Use Cases of high and full Driving Automation based on transport-related Experiences", in Transportation Research Procedia 41, 609-613. DOI: 10.1016/j.trpro.2019.09.108.

Mörner, M. von 2018. „Sammelverkehr mit autonomen Fahrzeugen im ländlichen Raum“, Dissertation, TU Darmstadt.

Ohnemus, M., und A. Perl 2016. „Shared Autonomous Vehicles: Catalyst of New Mobility for the Last Mile?“, in Build Environment (42) 4, 589-602.

Perkins, L., N. Dupuis und B. Rainwater 2018. „Autonomous Vehicle Pilots Across America - Municipal Action Guide“. Washington D.C.: National League of Cities - Center for City Solutions. www. nlc.org/sites/default/files/2018-10/AV\%20MAG\%20Web.pdf (19.8.2020).

Regional Transportation District 2019. „University of Denver Autonomous Vehicle Shuttle - Automated Driving Demonstration Grants“, 21.3.2019. Washington D.C. www.transportation.gov/ sites/dot.gov/files/docs/policy-initiatives/automated-vehicles/351416/69-university-denver.pdf (19.8.2020).

Rentschler, C., L. Herrmann, D. Kurth, W. Manz und M. Rumberg 2020. „Technische und rechtliche Systemgrenzen in der Routenplanung autonomer Shuttlebusse“, in: Neue Dimensionen der Mobilität. Technische und betriebswirtschaftliche Aspekte, hg. v. H. Proff. Wiesbaden: Gabler, 319-331. 
Röhrleef, M. 2017. „Autonomes Fahren: Himmel oder Hölle für den ÖPNV?“. https://bildungsservice. vcd.org/fileadmin/user_upload/Weiterbildung/Fachtagung_2016/Roehrleff_VCD_2050_autonomes_Fahren.pdf (7.1.2020).

Rollinger, W., und G. Amtmann 2009. Handbuch Öffentlicher Verkehr. Schwerpunkt Österreich, hg. v. ÖVG Österreichische Verkehrswissenschaftliche Gesellschaft, Arbeitskreis Öffentlicher Verkehr. Wien: Bohmann.

Rutanen, E., und V. Arffman 2017. „Autonomous robot bus experiments on public roads - SOHJOA Project report autumn 2017“, 11.12.2017. Download unter https://tinyurl.com/y567zhgb (19.8.2020).

Salonen, A. O., und N. Haavisto 2019. „Towards Autonomous Transportation. Passengers' Experiences, Perceptions and Feelings in a Driverless Shuttle Bus in Finland.“, in Sustainability (11) 588, $1-19$.

SBB (Schweizer Bundesbahnen) 2020. „MyShuttle Abschlussbericht“, 30.4.2020. Download unter www.astra.admin.ch/dam/astra/de/dokumente/abteilung_strassennetzeallgemein/sbb-myshuttle-abschlussbericht.pdf.download.pdf/SBB_MyShuttle_Abschlussbericht.pdf (7.6.2020).

Sinner, M. 2019. „Effects of the Autonomous Bus on the Railway System“, Dissertation, Institut für Verkehrsplanung und Transportsysteme (IVT), ETH Zürich.

Sinner, M., P. Khaligh und U. Weidmann 2018. „Consequences of automated transport systems as feeder services to rail: SBB fund for research into management in the field of transport", IVT Schriftenreihe Band 184. ETH Zürich. Download unter www.research-collection.ethz.ch/handle/20.500.11850/266025.

Sommer, C. 2018. „Neue Angebote für den Iändlichen Raum“, Präsentation im Rahmen der Veranstaltung „Zentralitäten 4.0 - Mittelzentren im Zeitalter der Digitalisierung?“, 22.11.2018, Kassel.

Soteropoulos, A., A. Stickler, V. Sodl, M. Berger, J. Dangschat, P. Pfaffenbichler, G. Emberger, E. Frankus, R. Braun, F. Schneider, S. Kaiser, H. Walkobinger und A. Mayerthaler 2019. „SAFiP Systemszenarien Automatisiertes Fahren in der Personenmobilität. Endbericht“, hg. v. Bundesministerium für Verkehr, Innovation und Technologie, Wien. https://projekte.ffg.at/anhang/5cee1b11a1eb7_SA-FiP_Ergebnisbericht.pdf (11.11.2020).

Weidmann, U., R. Dorbitz, H. Orth, M. Scherer und P. Spacek 2011. „Einsatzbereiche verschiedener Verkehrsmittel in Agglomerationen“, Forschungsauftrag SVI 2004/039 auf Antrag der Schweizerischen Vereinigung der Verkehrsingenieure und Verkehrsexperten (SVI). Bern: Eidgenössisches Departement für Umwelt, Verkehr, Energie und Kommunikation UVEK.

Wolf-Eberl, S., H. Koch, G. Estermann und A. Fürdös 2011. „Ohne eigenes Auto mobil - Ein Handbuch für Planung, Errichtung und Betrieb von Mikro-ÖV Systemen im ländlichen Raum“, Blue Globe Manual Mobilität 10/2011. In Zusammenarbeit mit dem Bundesministerium für Verkehr, Innovation und Technologie (bmvit) und im Auftrag des Klima- und Energiefonds. Wien. http://rdc.co.at/wpcontent/uploads/2017/11/Mikro_ÖV_Handbuch_publiziert_2011.pdf (19.8.2020).

Open Access Dieser Beitrag wird unter der Creative Commons Namensnennung 4.0 International Lizenz (http://creativecommons.org/licenses/by/4.0/deed.de) veröffentlicht, welche die Nutzung, Vervielfältigung, Bearbeitung, Verbreitung und Wiedergabe in jeglichem Medium und Format erlaubt, sofern Sie den/die ursprünglichen Autor(en) und die Quelle ordnungsgemäß nennen, einen Link zur Creative Commons Lizenz beifügen und angeben, ob Änderungen vorgenommen wurden.

Die in diesem Beitrag enthaltenen Bilder und sonstiges Drittmaterial unterliegen ebenfalls der genannten Creative Commons Lizenz, sofern sich aus der Abbildungslegende nichts anderes ergibt. Sofern das betreffende Material nicht unter der genannten Creative Commons Lizenz steht und die betreffende Handlung nicht nach gesetzlichen Vorschriften erlaubt ist, ist für die oben aufgeführten Weiterverwendungen des Materials die Einwilligung des jeweiligen Rechteinhabers einzuholen.

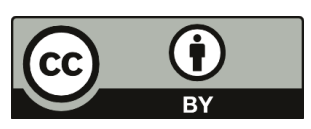

